\title{
Noncovalent interactions in high-performance liquid chromatography enantioseparations on polysaccharide-based chiral selectors
}

\author{
Paola Peluso Conceptualization; Data curation; Writing - original draft; review \& editing ${ }^{\mathrm{a}, *}$ \\ Victor Mamane Data curation; Writing - original draft; review \& editing ${ }^{\mathrm{b}}$, Roberto Dallocchio ${ }^{\mathrm{a}}$, \\ Alessandro Dessì ${ }^{a}$, Sergio Cossu Data curation; Writing - review \& editing ${ }^{c}$

\footnotetext{
${ }^{a}$ Istituto di Chimica Biomolecolare ICB, CNR, Sede secondaria di Sassari, Traversa La Crucca 3, Regione Baldinca, I-07100 Li Punti - Sassari, Italy

${ }^{\mathrm{b}}$ Institut de Chimie de Strasbourg, UMR CNR 7177, Equipe LASYROC, 1 rue Blaise Pascal, 67008 Strasbourg Cedex, France

' Dipartimento di Scienze Molecolari e Nanosistemi DSMN, Università Ca' Foscari Venezia, Via Torino 155, 30172 Mestre Venezia, Italy
}

\section{A R T ICLE INFO}

\section{Article history:}

Received 26 March 2020

Received in revised form 2 May 2020

Accepted 4 May 2020

Available online $\mathrm{xxx}$

\section{Keywords}

Chiral recognition

High-performance liquid-chromatography

Molecular modelling

Noncovalent interactions

Polysaccharide-based chiral stationary phases

\begin{abstract}
A B S T R A C T
Designed more than thirty years ago in order to improve and maximize the discrimination capability of native polysaccharides, cellulose- and amylose-based selectors have shown excellent and unequalled performances for the enantioseparation of chiral compounds. The successful story of these chiral selectors relies on a multi-site high-ordered chiral platform which is held up by intramolecular hydrogen bonds (HBs), and makes the polymer able to host and discriminate enantiomers. In this environment, both achiral and stereoselective intermolecular noncovalent interactions play a pivotal role, and HBs, halogen bonds (XBs), dipole-dipole, $\pi-\pi$ stacking, steric repulsive, and van der Waals interactions underlie adsorption process and formation of transient diastereomeric assemblies between the polymer and the enantiomer pair. In the last decades, advances in computational chemistry and spectroscopic techniques have improved knowledge of noncovalent interactions, contributing to decode their functions in chemical systems. Significantly, over time the growing interplay between experimental and theoretical approaches has contributed to unravel intermolecular forces underlying selector-selectand association and to understand recognition patterns. On this basis, this review summarizes seminal and representative studies dealing with noncovalent interactions that function in HPLC enantioseparations promoted by cellulose benzoates and phenylcarbamates of amylose and cellulose. The importance of integrating theoretical and experimental approaches to profile mechanisms and interaction patterns is highlighted by discussing focused case studies. In particular, the advantageous utilization of electrostatic potential (V) analysis and molecular dynamics (MD) simulations in this field is evidenced. A systematic compilation of all published literature has not been attempted.
\end{abstract}

(C) 2020

\section{Introduction}

Noncovalent interactions were first described by J. D. van der Waals in the $19^{\text {th }}$ century, his studies providing a notable and influential contribution to the comprehension of molecular cohesion and intermolecular forces [1]. During the time, the concept has been defined by several authors focusing on the inherent difference between covalent and noncovalent bonds [2-4]. In both cases, bonds originate from a primary interaction between atoms and molecules which leads to the formation of a covalent bond and a new molecule, or a noncovalent bond and a new molecular assembly [3]. On the basis of their essential function of connecting atoms and molecules, noncovalent interactions control molecular binding and recognition, allowing for the development of diverse applications of supramolecular chemistry [4]. Indeed, depending

\footnotetext{
* Corresponding author. Tel.: + 390792841218

E-mail address: paola.peluso@cnr.it (P. Peluso)
}

on the structural features of the interacting parts and medium properties, ionic interactions, hydrogen bond (HB), $\sigma$ - and $\pi$-hole bonds, anionand cation- $\pi, \pi-\pi$ stacking, dipole-dipole, hydrophobic, steric repulsive, and van der Waals (vdW) interactions can occur at intra- and intermolecular level, originating and regulating formation, properties, functions and applications of supramolecular complexes, assemblies and clusters in gas, liquid and solid phase [5].

In the last decades, huge progresses have been made in the understanding [6] and measurement of molecular interactions [7]. Significantly, advancements in crystal engineering, analytical methods and computational chemistry have allowed for a deeper profiling of noncovalent interaction nature and functions $[3,8,9]$. As a consequence of the recognized relevance of noncovalent bonds in chemistry, physics, and biology, the International Union of Pure and Applied Chemistry issued focused recommendations on hydrogen [10], halogen [11], and chalcogen [12] bonds.

Noncovalent interactions also play a key role in enantioseparation science, where they are the essential elements of the code by which chiral selector and selectand enantiomers connect to each other to per- 
form the enantiomer distinction $[13,14]$. In this field, HPLC enantioseparations on polysaccharide-based chiral stationary phases (CSPs) still remain the most used for the resolution of racemic mixtures, these selectors being extremely popular due to their versatility and separation performances [15-18]. Indeed, the complex structure of the polysaccharide-based selectors represents a multivalent adaptable chiral platform which contains different recognition sites with functions as both Lewis bases and acids [19]. A dynamic and reversible process moving the enantiomers from the mobile phase (MP) to the chiral polymer surface enables the formation of two transient diastereomeric complexes, which are characterized by different free energy $\left(\Delta \Delta G^{\circ}\right)$ and lifetime. Consequently, enantiomers are eluted outside the chiral column in different times, with an enantiomer elution order (EEO) dependent on the preference of the selector toward one of two enantiomeric three-dimensional structures. At molecular level, the distinction process is based on the ability of the polysaccharide-based selector to recognize the enantiomers of the analyte by means of HBs and halogen bonds (XBs), dipole-dipole and $\pi-\pi$ stacking, vdW and hydrophobic forces, and steric repulsion, the strength of these interactions being dependent on selector and analyte structure, and MP. Indeed, on the basis of its structure and polarity, the MP can favour or disfavour selector-analyte interactions through solvation effects. Thus, selector, analyte, and MP determine noncovalent interaction patterns underlying chiral recognition and affect $\Delta \Delta G^{\circ}$ and its enthalpic and entropic terms, according to the Gibbs-Helmholtz equation (1)

$$
\Delta \Delta G^{\circ}=\Delta \Delta H^{\circ}--\mathrm{T} \Delta \Delta S^{\circ}
$$

This structural complexity, and the occurrence of multiple intermolecular forces which act between selector and selectand in a solvated environment, represent the key of the success of polysaccharide-based CSPs. On the other hand, the high level of intricacy makes the identification of noncovalent interactions underlying chiral recognition mechanisms a challenging and still open issue $[20,21]$. To tackle this task, integrating theoretical and experimental studies represents a modern and useful approach to profile the enantiodistinction process [22,23]. The interplay between chromatographic, thermodynamic, extrathermodynamic, spectroscopic, X-ray diffraction (XRD), and computer-aided studies has been contributing more and more to develop structure-retention and structure-selectivity models with adequate degrees of reliability $[2,24,25]$. In the last decade, these combined approaches have been fruitfully applied to explore chiral recognition mechanisms occurring on polysaccharide-based and other chiral selectors such as cyclodextrins, cyclofructans, macrocyclic glycopeptides, proteins, and small chiral selectors $[20,21,25]$. Among computational approaches, molecular dynamics (MD) simulations [22,23] have proven to be a powerful tool furnishing intriguing details on enantiodiscrimination at a microscopic level. Moreover, recently electrostatic potential $(V)$ analysis allowed for identifying and studying XB and other interactions in HPLC environment [26-28].

Given this context, noncovalent interaction functions in enantioseparation processes promoted by cellulose benzoates and amylose and cellulose phenylcarbamates in HPLC environment are described herein. First, some introductory concepts on selectors, noncovalent interactions, and approaches to study HPLC recognition mechanisms are summarized. Then, seminal and more recent studies are discussed, which were performed a) to explore and model the high-ordered structure of polysaccharide-based selectors, and b) to evaluate the contribution of intermolecular noncovalent contacts to analyte adsorption and discrimination. Since excellent reviews on polysaccharide-based CSPs have been recently published $[17,18]$, aspects concerning applications, enantioseparation method development, instrumental issues and immobilization technologies are not discussed in this article.

\section{Brief description of amylose- and cellulose-based chiral stationary phases}

Cellulose benzoates and phenylcarbamates of cellulose and amylose (Fig. 1), bearing one or two electron-donor or electron-withdrawing substituents on the aryl ring, were designed and prepared by Okamo-

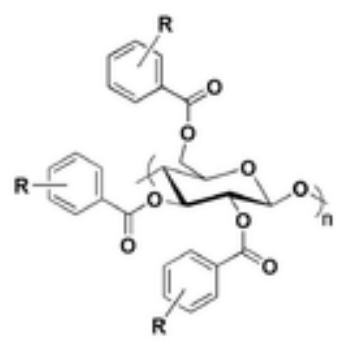

cellulose tribenzoates (CTBs)

\begin{tabular}{|l|l|}
\hline Column tradename & $R$ \\
\hline Chiralcel OB' & H \\
\hline $\begin{array}{l}\text { Chiralcel OJ } \\
\text { Lux Cellulose-3 }\end{array}$ & $4-3^{\circ}$ \\
\hline
\end{tabular}

- Chiral Technologies (Daicel) b Phenomenex

'other tradenames (suppliers):

CelluCoat (EKA), RegisCen

(Regis Technologies), Nucleocel delta (Macherey\&Nagel), Eurocel 01 (Knaver).

${ }^{o}$ other tradenames (suppliers): AmyCoat (EKA), RegisPack

(Regis Technologies), Nucleocel alfa (Macherey\&Nagel), Europak 01 (Knaver).

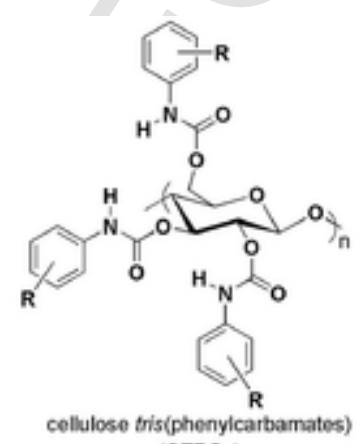

(CTPCs)

\begin{tabular}{|c|c|}
\hline Column tradename & $R$ \\
\hline Chiralcel OC & $\mathrm{H}$ \\
\hline Chiralcel OG ${ }^{a}$ & $4-\mathrm{Me}$ \\
\hline $\begin{array}{l}\text { Chiralcel OD.c } \\
\text { Lux Cellulose- } 1^{b} \\
\text { Chiralpak IB (immobilizeof) }\end{array}$ & 3,5-diMe \\
\hline Chiralcel OF* & $4-\mathrm{Cl}$ \\
\hline $\begin{array}{l}\text { Chiralcel OX } \\
\text { Lux Cellulose-4 }\end{array}$ & 4-Cl-3-Me \\
\hline $\begin{array}{l}\text { Chiralcel OZ" } \\
\text { Lux Cellulose-2 }\end{array}$ & 3-Cl-4-Me \\
\hline $\begin{array}{l}\text { Chiralpak IC (imm.) } \\
\text { Lux i-Cellulose-5 (imm.) }\end{array}$ & 3,5-diCl \\
\hline
\end{tabular}

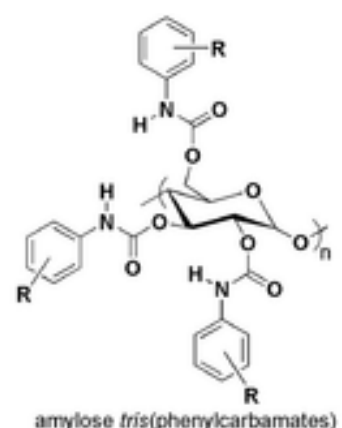

(ATPCs)

\begin{tabular}{|c|c|}
\hline Column tradename & $R$ \\
\hline $\begin{array}{l}\text { Chiralpak } A D^{\text {ed }} \\
\text { Lux Amylose-1 } 1^{\circ} \\
\text { Chiralpak } \mid \mathrm{A}^{a} \text { (imm.) } \\
\text { Lux i-Amylose-16 (imm.) }\end{array}$ & 3,5-diMe \\
\hline Chiralpak ID $(\mathrm{imm}$ ) & 3- $\mathrm{Cl}$ \\
\hline $\begin{array}{l}\text { Chiralpak AY" } \\
\text { Lux Amylose-2 } 2^{\circ}\end{array}$ & 5. $\mathrm{Cl}-2-\mathrm{Me}$ \\
\hline 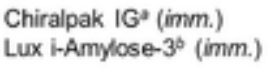 & 3. $\mathrm{Cl}-5 \cdot \mathrm{Me}$ \\
\hline $\begin{array}{l}\text { Chiralpak AZ } \\
\text { Chiralpak IF* (imm.) }\end{array}$ & 3. $\mathrm{Cl}-4-\mathrm{Me}$ \\
\hline Chiralpak IE (imm.) & 3,5-diCl \\
\hline
\end{tabular}

Fig. 1. Structures and column tradenames of cellulose tribenzoates, and amylose and cellulose tris(phenylcarbamate)s which are commercialized as CSPs. 
to's group in 1980s [29-33] in order to improve and maximize the discrimination capability of native polysaccharides. Later, in 1990s a new series of polymers containing substituents of different nature was developed [34-36], and over time some of these polymeric selectors have shown excellent and unequalled performances for the enantioseparation of chiral compounds $[15,17]$. On these bases, several chiral columns are today commercially available, which contain silica supports functionalized with the polymeric selector that is covalently linked or physically adsorbed (Fig. 1).

Actually, several polysaccharides such as cellulose, amylose, inulin, curdlan, chitin, chitosan, xylan and dextran were considered [29], but to date only cellulose and amylose derivatives have shown suitable per- formances to be commercialized. Moreover, despite the fact that both chitosan and chitin derivatives showed promising performances for enantioseparation $[37,38]$, no theoretical exploration has been attempted so far in order to understand their recognition mechanisms towards enantiomers [21].

As depicted in Fig. 2 for the amylose tris(3,5-dimethylphenylcarbamate) (ADMPC) as a representative example [39], in amylose- and cellulose-based selectors, the polysaccharide backbone is the essential element because conformational chirality depends on the peculiar helical twist generated by specific glycosidic linkages in cellulose $(\beta-1,4)$ and amylose $(\alpha-1,4)$ polymeric chains. The native polysaccharide is modified at two different levels through $a$ ) a polar layer containing carba- (a)<smiles>Cc1cc(C)cc(NC(=O)Nc2cc(C)cc(C)c2)c1</smiles>

amylose tris(3,5-dimethylphenylcarbamate) (c)

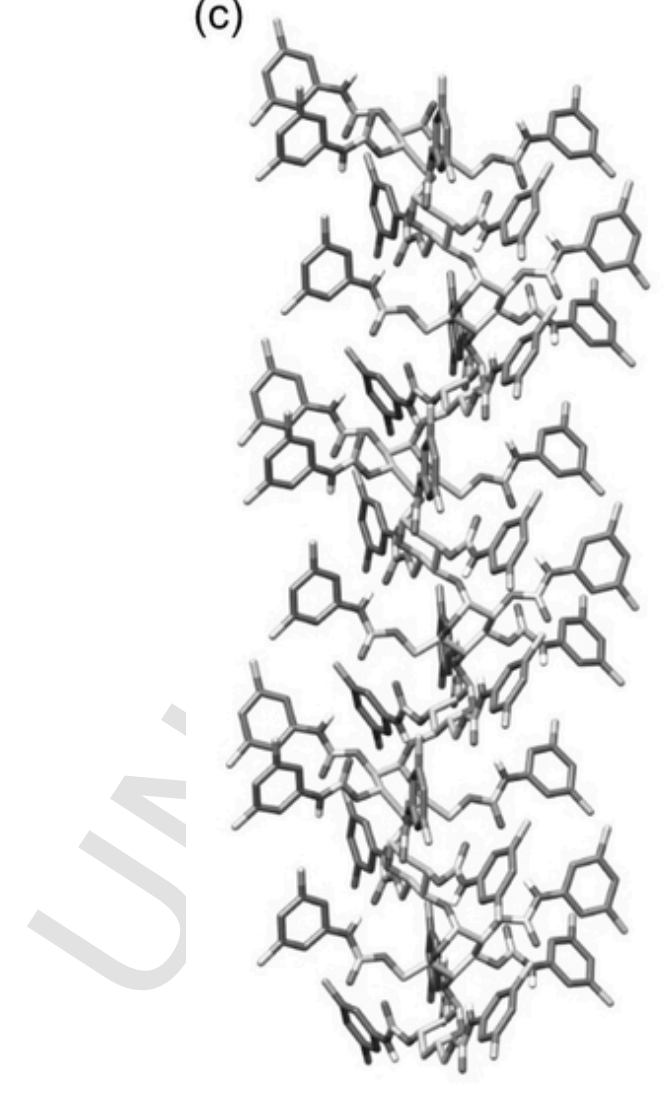

(b)

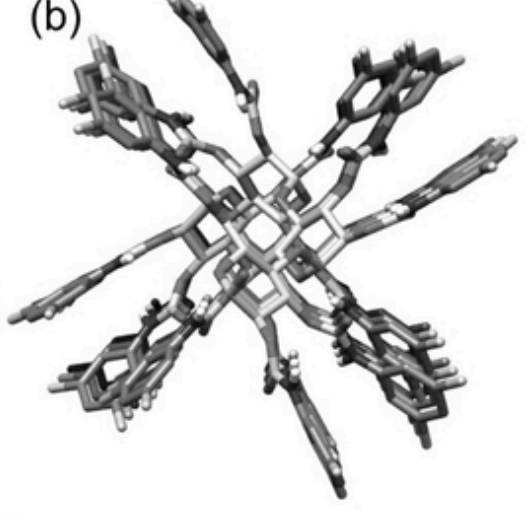

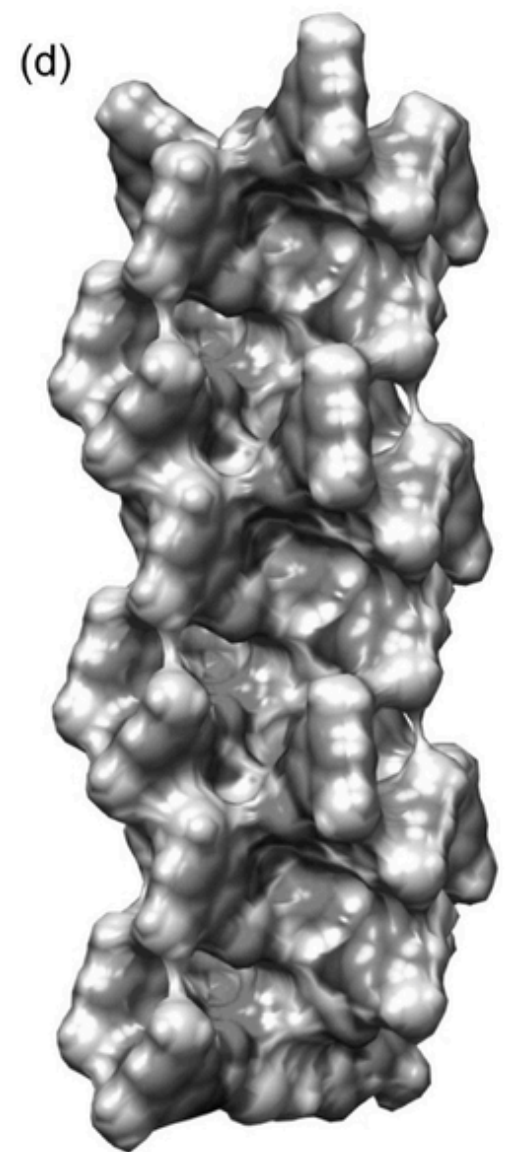

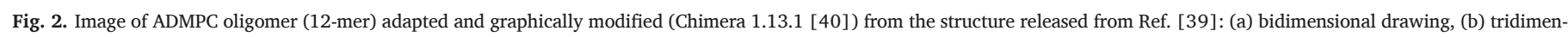
sional, viewpoint along the chain axis, (c) viewpoint perpendicular to the chain axis, (d) electron density surface. Colours: aryl group (green), $\mathrm{N}$-H (blue), $\mathrm{C}=\mathrm{O}$ (red). 
mate or benzoate groups which are close to the polymer backbone (N-H coloured in blue, $\mathrm{C}=\mathrm{O}$ coloured in red), and able to exert polar interactions, and $b$ ) a hydrophobic layer containing substituted aromatic rings (coloured in green), which are located at the periphery of the polymer, and able to exert $\pi-\pi$ interactions (Fig. 2a). The result of this derivatization is the radial expansion of the polymer in a direction perpendicular to the backbone axis (Fig. 2b), and the transfer of the chiral information from the molecular to a conformational level to generate a chiral polymeric groove (Fig. 2c,d) which is able to host a chiral molecule, and recognize, and differentiate its enantiomers.

Among several other atoms or groups evaluated as substituents, methyl and chlorine proved to be the best electronic modulators inside the polymeric system. Consequently, the most commercialized selectors contain methyl, chlorine or both as substituents, which are the key to the different selectivity of the corresponding CSPs. Indeed, the electronic properties of the polar layer (carbamate or benzoate) and its ability to exert polar interactions are tuned by changing type and position of both methyl- and chloro-substituents onto the terminal aromatic ring [16,34-36]. Thus, a multitude of noncovalent interactions can potentially occur into the groove but, actually, only some of them act to recognize the enantiomers of a given chiral analyte, depending on its particular structure, size and shape which is the sum of geometry and electronic distribution. In this regard, it is worth mentioning that noncovalent interactions operating in this class of polymers can be also ex-

Table 1

Type, direction, and angular dependence of noncovalent interactions working in polysaccharide-based CSPs.

\begin{tabular}{|c|c|c|}
\hline Type & Direction & Angular dependence \\
\hline Hydrogen bond (HB) & Attractive & Directional \\
\hline Halogen bond (XB) & Attractive & Directional \\
\hline$\pi-\pi$ interaction & Attractive & Directional \\
\hline Dipole-dipole & Attractive & Directional \\
\hline van der Waals induction force ${ }^{a}$ & Attractive & Directional \\
\hline London dispersion force ${ }^{b}$ & Attractive & Non-directional \\
\hline Hydrophobic interaction & Attractive & Non-directional \\
\hline Steric hindrance & Repulsive & Non-directional \\
\hline
\end{tabular}

a permanent dipole-induced dipole

b induced dipole-instantaneous dipole

(a)

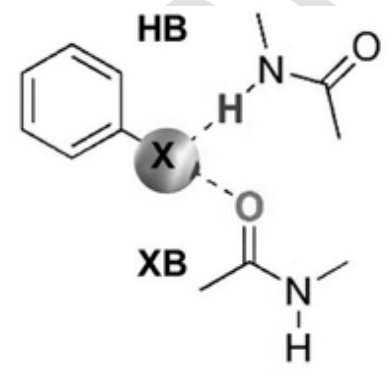

(b)

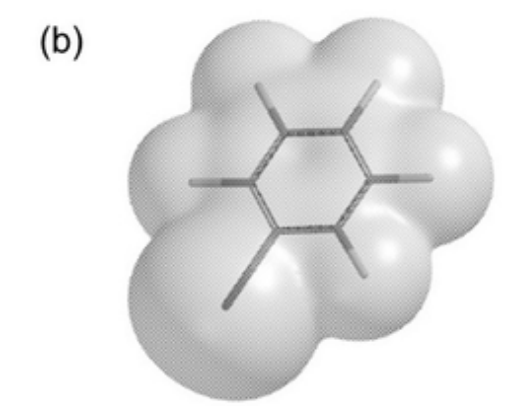

Fig. 3. Scheme of HBs between analytes and carbamate moiety of polysaccharide-based selectors.

ploited to discriminate non-enantiomeric compounds which are difficult to separate with conventional achiral materials [41-43]. In addition, polysaccharide-based CSPs have been successfully used for preparative enantioseparation due to their high loading capacity which increases the productivity of the separation process [44]. Indeed, the high density of chiral selector molecules per surface unit allows for an elevated simultaneous number of noncovalent interactions acting to discriminate analyte molecules [45]. Thus, the efficient network of noncovalent interactions promoted by cellulose- and amylose-based CSPs lends them the highest loading capacity among all chiral materials used for preparative applications.

\section{Noncovalent interactions in polysaccharide-based selectors}

Noncovalent interactions which can drive association of enantiomers and polysaccharide-based selectors are summarized in Table $1[46,47]$.

HBs occur either between positively polarized hydrogen atoms of carbamates $\mathrm{N}-\underline{\mathrm{H}}$ (selector) as $\mathrm{HB}$ donors and negatively polarized $\mathrm{HB}$ acceptors of analytes, either between oxygen atoms of benzoate or carbamate $\mathrm{C}=\mathrm{O}$ (selector) as $\mathrm{HB}$ acceptors and positively polarized $\mathrm{HB}$ donors of analytes (Fig. 3), with distances ranging typically from 2.8 to $3.2 \AA[39,48]$.

$\mathrm{XBs}$ have been found in polysaccharide-based selectors occurring between the carbamate $\mathrm{C}=\underline{\mathrm{O}}$ and a halogen atom of analytes which behaves like Lewis acid through the region of lower electron density called $\sigma$-hole (Fig. 4a), and located on the elongation of C-X bond $[27,49]$. The strength of the $\mathrm{XB}$ depends on halogen type following the order $\mathrm{I}>\mathrm{Br}>\mathrm{Cl}$, and typical distances have been observed in the range 2.8-3.5 $\AA[27,50]$. On the other hand, halogen can behave as $\mathrm{HB}$ acceptor toward the carbamate hydrogens $\mathrm{N}-\mathrm{H}$ by means of the
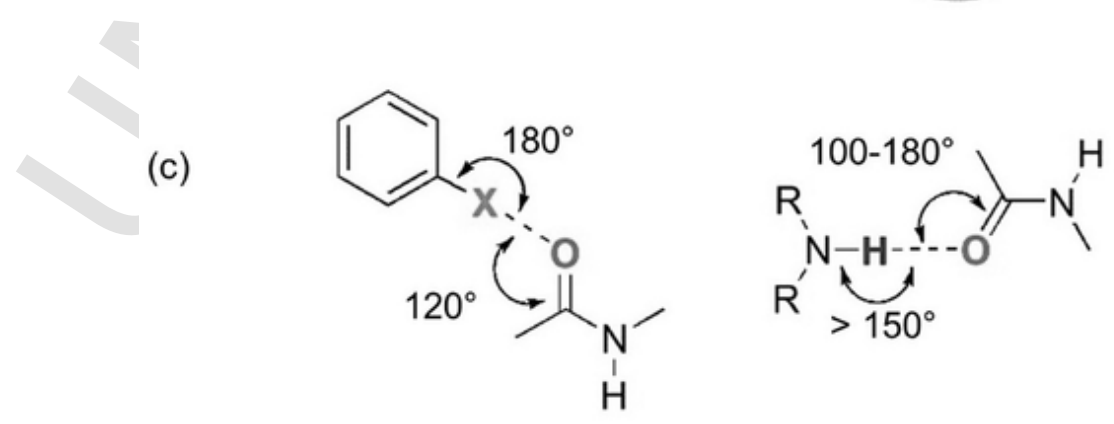

Fig. 4. (a) General scheme of $\mathrm{XB}$ and $\mathrm{HB}$ on halogen site; (b) hydrophobic surface of iodopentafluorobenzene; (c) typical angular features of XB and HB. 
higher electron density region located around the $\sigma$-hole, as well as hydrophobic site, specially in polar solvents (Fig. 4b). Both HB and XB are directional interactions with a strong angular preference (Fig. 4c).

$\pi-\pi$ Interactions are attractive and involve $\pi$-clouds centred on aromatic rings belonging to both analyte and polysaccharide structures. Like HBs, these interactions are also based on complementarity, therefore they occur between a negatively polarized $\pi$-cloud as a $\pi$-donor ( $\pi$-basic ring) and a positively polarised $\pi$-cloud as a $\pi$-acceptor ( $\pi$-acid ring). Dipole-dipole interactions are attractive and involve regions having a dipole moment. Carbamate and benzoate carbonyls represent the main dipolar sites in the polysaccharide-based CSPs. Induction and dispersion forces are the weakest noncovalent interactions. However, these forces contribute to the hydrophobic effect playing a significant role in reversed-phase elution mode and some entropy-driven processes [46]. Finally, noncovalent interactions can behave in a repulsive manner, short-range repulsion due to steric hindrance being originated by overlap of the electron distribution. It affects discrimination due to the fact that atoms, functional groups and molecules occupy a space which cannot be occupied by other molecular entities.

Several other features characterize noncovalent interaction patterns in multivalent polysaccharide-based selectors: i) phenomena of positive and negative cooperativity can limit the additivity of single interactions $[4,8,48]$; ii) many typical interactions can be considered multipoint interactions in their nature such as dipole-dipole and $\pi-\pi$ interactions [25]; iii) conformational features of the interacting partners affect noncovalent interaction efficacy; iv) when a molecule interacts with another molecule, its electronic density is perturbed to some extent by the electric field of the other molecule [51]. This induced polarization may significantly influence each further interactive contact, affecting interaction additivity also in this case.

In addition, type of operating noncovalent interactions can be significantly modulated by changing the MP. In general, three elution modes can be exploited, which are the normal phase (NP), the polar organic (PO), and the reversed-phase (RP) [52,53]. Under NPLC, the interaction capability of the CSP is governed by polar interactions. Using PO conditions, namely acetonitrile or alcohols, allows to properly modulate the strength of polar and hydrophobic interactions which increase and decrease, respectively, following the order methanol $\rightarrow$ ethanol $\rightarrow$ 2-propanol $[54,55]$. Solvents of medium polarity (nonstandard solvents) such as dichloromethane, tetrahydrofuran, ethyl acetate, and methyltert-butyl ether can be used on immobilized selectors [56], whereas these solvents cannot be used with coated polymers. Under RP elution conditions, namely using aqueous MPs $[57,58]$, the polymer can behave as a non-polar CSP and its interaction capability can be governed by hydrophobic interactions. However, as polysaccharide-based CSP contain carbamate and ester substructures along with hydrophobic regions, hydrophilic interactions can also occur with water-containing MPs. Therefore, based on analyte features, CSP structure, and water-containing MP composition, polysaccharide-based CSPs may behave as hydrophilic interaction liquid chromatography (HILIC)-like stationary phases, showing behaviour opposite to the RP mode due to a fine balance between HBs and hydrophobic contacts as the content of water changes [18,59-61].

As the EEO describes the preference of the selector towards one of two enantiomeric three-dimensional structures, factors influencing discrimination mechanism at molecular level such as CSP, MP type and composition, and temperature, can also impact EEO. Significantly, phenomena of EEO reversal dependent on these factors disclose that changes in discrimination mechanism occur. In this regard, it is worth mentioning that interesting examples of EEO reversal dependent on nature and content of PO modifiers and additives in the MP and on temperature were described in literature [53,62-65]. In particular, EEO reversal dependent on the content of water in aqueous-organic MP has been reported revealing the dual behaviour of the CSP as HILIC-type and RP sorbent [61].
In this complex scenario, information on chiral recognition mechanisms can be available by using orthogonal techniques which can furnish complementary evidences on the enantioseparation event at macroscopic and microscopic level. Most studies started from the experimental chromatographic evidences, and empirical recognition models could be derived by evaluation of the effect of MP nature, structure of polysaccharide derivative and analyte on retention and selectivity [30,34,66,67]. Thermodynamic quantities estimated by van't Hoff analysis also provide meaningful information at macroscopic level in order to derive the enthalpic and entropic contributions to the enantiodiscrimination [18]. Nevertheless, van't Hoff analysis does not allow for determining individually achiral and chiral features of enantioseparation and their actual ratio in the discrimination process $[68,69]$. Going beyond the boundaries of pure chromatographic studies, spectroscopic methods [39,70], XRD [71] and molecular modelling [22,23,39,72,73] have been fruitfully used for the purpose along with extrathermodynamic approaches such as entropy-enthalpy compensation (EEC), linear free energy relationship (LFER), and linear solvation energy relationships (LSER) [25,74-76]. Among all possible approaches, significant information on noncovalent interactions at microscopic level have been gained by spectroscopic and computational methods [21-24].

Nowadays, molecular modelling has become more and more faithful to simulate enantiomer distinction, in particular, due to the availability of molecular mechanics (MM) methods adaptable to the study of large systems, the improvements of software and hardware tools, and the availability of friendly computational platforms. In this context, docking, MD, and $V$ analysis provide important information to visualize analyte-polymer association and to identify type, topology and geometrical parameters of noncovalent interactions underlying complex formation. Developed at the beginning to explore and understand protein-ligand contacts in medicinal and pharmacological chemistry [48,77], these techniques have also found fruitful application in other fields such as chiral chromatograpy. On one hand, docking presents a very low computational cost, allowing exploration of a vast conformational space in a short time. On the other hand, a problem emerges from the poor flexibility of the modelled polymer which is not permitted to adjust its conformation upon analyte binding. On the contrary, MD simulations are time-consuming but they treat simulation in a flexible way, and the effect of MP can be properly accounted for by parametrizing the solvent explicitly. On this basis, a useful multistep approach consists in the following sequence: a) docking for a preliminary exploration about polysaccharide-enantiomers binding possibilities, b) MD simulation in order to account the dynamic nature of enantioseparation, c) refinement of low-energy selector/enantiomer complexes at quantum mechanics (QM) level in order to obtain accurate energetic information. However, interesting energetic information could be also extracted from MD trajectory data set. Indeed, during MD production the molecular system is free to run for a period of time and the process is iterated for thousands of steps in order to bring the system to an equilibrium state, saving all the information about the atomic positions, velocities, and other variables as a function of time. The set of data emerging from the MD experiment is the trajectory which describes coordinates and energies of the chiral partners in the system and their variation with time. In most studies focused on cellulose- and amylose-based derivatives as selectors $[70,78-80]$, the interaction energy $\left(E_{\text {int }}\right)$ between enantiomer and selector is calculated on the basis of the energies of the selector-enantiomer complex, the selector and the enantiomer (eq. 2)

$\begin{aligned} E_{\text {int }}= & E_{\text {total }}--E_{\text {enantiomer }}- \\ & -E_{\text {polysaccharide-based selector }}\end{aligned}$

where the $E_{\text {int }}$ term derived from the contributions of the vdW and the electrostatic (es) interaction terms eq. 3). The term $\mathrm{E}_{\mathrm{vdW}}$ in turn is composed of repulsive (rep) and dispersive (disp) energy (eq. 4,(6)

$E_{\text {int }}=E_{\mathrm{es}}+E_{\mathrm{vdW}}$ 
$E_{\mathrm{vdW}}=E_{\mathrm{rep}}+E_{\mathrm{disp}}$

where

$E_{\mathrm{es}}=\sum_{i, j>i}\left(q_{i} q_{j}\right) /\left(4 \pi \varepsilon_{0} r_{i j}\right)$

$E_{\mathrm{vdW}}=\sum_{i, j>i}\left(A_{i j} / r_{i j}^{12}-B_{i j} / r_{i j}^{6}\right)$

In equations $(5,6), q_{i}$ and $q_{j}$ represent electric charge of atoms $i$ and $j$, respectively, $\varepsilon_{0}$ is the dielectric constant, and $r_{i j}$ is the interatomic distance computed from the Cartesian coordinates. The parameters $A_{i j}$ and $B_{i j}$ are repulsive and attractive terms dependent on outer shell electrons and atom polarizabilities, respectively. The interaction energy values calculated for each selector-enantiomer complex allow for gaining information to rationalize the experimental EEO by visualizing the molecular reasons of the stereopreference of the selector toward one of the two enantiomers and involved noncovalent interactions.

Recently, $V$ analysis proved to be very promising to gain insights on noncovalent interaction function in polysaccharide-based CSPs by evaluating the electron density on molecular regions involved in the noncovalent contacts $[26,28,79]$. In fact, as the enantioseparation is a phenomenon involving surface contacts between selector and analyte, the $V$ analysis of the interacting partners in their unperturbed state can be advantageously used to predict and rationalize noncovalent interaction strength and direction by mapping $V$ on electron density isosurfaces and generating $V$ isosurfaces $\left(V_{S}\right)$, the most used isodensity contours being 0.001 or 0.002 au [81]. Given a molecule, the $V(\mathbf{r})$ at each point $\mathbf{r}$ in the surrounding space, is created by each nucleus (first positive term) and electron (second negative term) of the molecule and given by equation 7

$V(\mathrm{r})=\sum_{A} \frac{\mathrm{Z}_{A}}{\mathrm{R}_{A}-\mathbf{r}}-\int \frac{\rho(\mathbf{r}) \mathrm{d} \mathbf{r}^{\prime}}{\left|\mathbf{r}^{\prime}-\mathbf{r}\right|}$

where $Z_{A}$ is the charge on nucleus A located at $R_{A}$, and $\rho(\mathbf{r})$ is the electron density function. Thus, the sign of $V_{S}$ may be positive or negative depending on the dominant contribution, which is positive and negative from nuclei and electrons, respectively. The interesting feature of $V$ is that it is a real physical property, and the evaluation of its variations on the molecular isosurfaces accounts for the shape of the molecules which is the sum of geometry and electronic distribution. Moreover, $V$ gives information on specific regions of the molecules, such as lone pairs and $\pi$-clouds. In particular, regions with high (nucleophile) and low (electrophile) electron density can be identified by the minima $\left(V_{\mathrm{S} \text {,min }}\right)$ and maxima $\left(V_{\mathrm{S} \text {,max }}\right)$ values of $V_{\mathrm{S}}$, respectively. Given the enantiophore concept [82], calculating $V_{\mathrm{S}}$ allows for achieving important information on the 3D-shape of the enantiophore systems, on site geometry and expected directionality of the noncovalent interactions potentially involved in the enantiodistinction process. As representative examples, in Figure 5 calculated $V_{\mathrm{S}}$ of DFT energy-minimized structures of Tröger base (1) and trans-stilbene oxide (2) are reported [26]. In this case, a more negative $V_{\mathrm{S} \text {,min }}$ value was calculated on the nitrogen surface in $1\left(V_{\mathrm{S}, \min }(\mathrm{N})=-151.2 \mathrm{~kJ} / \mathrm{mol}\right)$ with respect to $V_{\mathrm{S}, \min }$ of the oxygen in $2\left(V_{\mathrm{S}, \min }(\mathrm{O})=-141.0 \mathrm{~kJ} / \mathrm{mol}\right)$. Compound 1 showing higher retention $\left(k_{1}=1.61\right)$ than $2\left(k_{1}=1.04\right)$ on cellulose tris(3,5-dimethylphenylcarbamate) (CDMPC) under NP elution conditions, the $V$ analysis confirmed that a HB between the HB acceptors of the analytes and the polymer contributes to retention of both $\mathbf{1}$ and $\mathbf{2}$ on CDMPC.

Moreover, even if $V_{S}$ is an achiral descriptor, it can account for those interactions governing enantioseparation because individual intermolecular interactions between the enantiomers and the CSP are achiral [76], while they differentiate the enantiomers on the basis of stereoselective cooperative patterns.

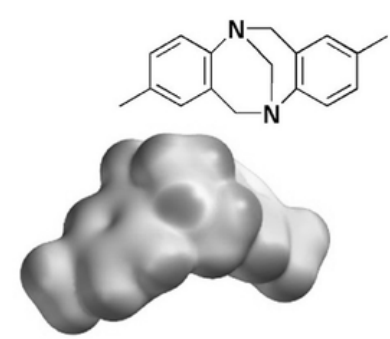

1

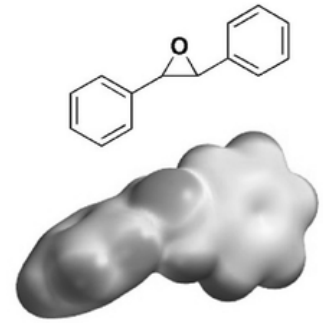

2
Fig. 5. $V_{\mathrm{S}}$ calculated for compound $1\left(V_{\mathrm{S}, \min }(\mathrm{N})=-151.2 \mathrm{~kJ} / \mathrm{mol}\right)$ and $2\left(V_{\mathrm{S}, \min }(\mathrm{O})=\right.$ $-141.0 \mathrm{~kJ} / \mathrm{mol}$ ) employing the DFT method with the B3LYP functional and the $6-31 \mathrm{G}^{* *}$ as basis set. Colours roward red depict negative potential, while colours toward blue depict positive potential and colours in between (orange, yellow, green) depict intermediate values of potential.

Another advantage of using $V$ is that it is less sensitive to the choice of basis set and computational methods than the calculation of interaction energy $[24,83]$. On this basis, even Hartree-Fock (HF) procedure can be used for larger molecules to profile trends and correlate with experimental data such as retention and selectivity values. In this regard, we recently found that correlation between $V_{\mathrm{S} \text {,max }}$ values calculated for a series of halogenated analytes and both retention and selectivity is not very sensitive to functional (B3LYP vs. M06-2X) and basis set (6-311G* vs. def2-QZVP) [24].

Descriptors related to $V$ and molecular surface properties [84] have been used in some quantitative structure-property relationships (QSPR) approaches such as 3D-QSPR comparative molecular field analysis (CoMFA) and comparative molecular similarity index analysis (CoMSIA). However, applications of these methodologies have been reported for polysaccharide derivatives $[85,86]$ in smaller degree compared to low-molecular weight selectors [87-89].

\section{Intra- and intermolecular noncovalent interactions}

From the first studies reported by Ichida [90] and Okamoto [29-33] describing the utilization of polysaccharides derivatives as chiral selectors for enantiomer separation, three main features emerged:

i) cellulose benzoates and phenylcarbamates of amylose and cellulose showed high degree of chiral recognition toward several classes of racemates [90,29,32];

ii) the high-ordered structure of polysaccharides appeared crucial for effective chiral discrimination, and the chiral recognition ability increased as the conformational regularity of the polysaccharide-derivative [19]. In particular, the phenylcarbamate derivatization favoured conformational regularity, and polysaccharide phenylcarbamate-based CSPs were able to enantioseparate Tröger base (1) and trans-stilbene oxide (2) used as test probes. On the contrary, phenylcarbamate of $\beta$-cyclodextrin adsorbed on silica gel as chiral stationary phase produced no enantioseparation for compounds 1 and 2, using $n$-hexane/2-propanol 99:1 as MP [29]. In addition, Okamoto and co-workers also used starch in place of amylose as chiral support [32]. This material consists of $20-25 \%$ of amylose and $75-80 \%$ of branched amylopectin. In this study, starch tris(3,5-dimethylphenylcarbamate) supported on silica gel exhibited lower chiral recognition ability compared to ADMPC as CSP, this result being probably due to the detrimental impact of branched amylopectin on the conformational regularity of the polymeric material.

iii) HBs and dipole-dipole interactions between the stationary phase and racemic compounds played an important role for chiral discrimination.

Therefore, two series of noncovalent forces control functions and patterns in polysaccharide based CSPs: a) intramolecular HBs 
which maintain the high-ordered structure of the polymer, and b) intermolecular noncovalent interactions which control selector-selectand association.

\subsection{Intramolecular hydrogen bonds and high-ordered structure of polysaccharide-based selectors}

In 1987, Vogt and Zugenmaier reported the X-ray analysis of a CTPC fibre which featured as a left-handed threefold 3/2 helix (Fig. 6a,b) [91]. This high-ordered structure is characterized by dihedral angles at the glycosyl linkages defined by $\mathrm{H}_{1}-\mathrm{C}_{1}-\mathrm{O}-\mathrm{C}_{4},(\phi)$ and $\mathrm{H}_{4},-\mathrm{C}_{4},-\mathrm{O}-\mathrm{C}_{1}(\psi)$ equal to $60^{\circ}$ and $0^{\circ}$, respectively (Fig. 6c).

Moreover, the side-groups at the 2- and 3-positions and that at the 6-position of the adjacent glucose unit are close to each other, with $s$-trans conformation between the carbonyl carbon and the ether oxygen on the side chain. Interestingly, a distance of $2.724 \AA$ was observed between the N- $\underline{H}$ proton and the carbonyl oxygen, a value which is typical for HBs (Fig. 6d). The same left-handed $3 / 2$ helical motif was also reported for cellulose tris(4-chlorophenylcarbamate) [92] by means of $\mathrm{X}$-ray analysis.

Computational models of polysaccharide derivative oligomers have been constructed by several groups over time as virtual platforms to perform MM energy minimization of selector-analyte complexes either by docking or by MD simulations of the selector-selectand association. For cellulose-based CSPs, the first model was built by Okamoto's group on the basis of known crystallographic structure provided by Vogt and Zugenmaier for CTPC [91]. In this regard, it was reported by light scattering and viscometry analysis that CTPC may mantain a helical structure also in solution [93]. On this basis, the computational model of CTPC [72] was built by means of a protocol consisting of a) full energy minimization of one unit of CTPC containing MeO groups at the terminal 1- and 4- positions, and b) construction of an octamer (8-mer) formed by the optimized units, assigning to the glycosyl dihedral angles the values derived from the X-ray structure reported by Vogt and $\mathrm{Zu}-$

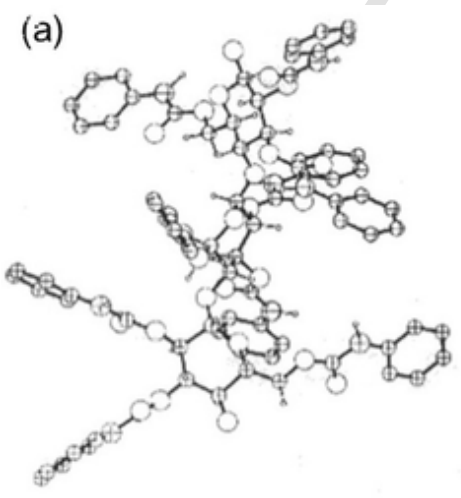

(c)

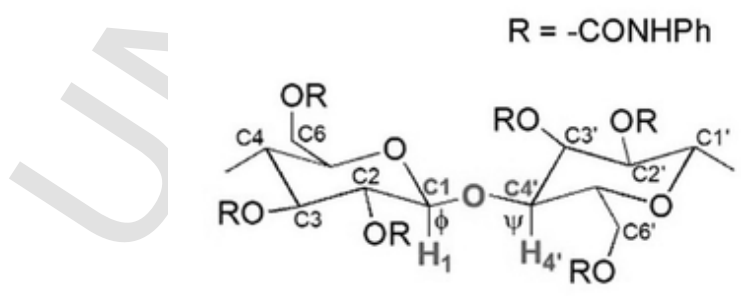

genmaier [91]. On this basis, a structure similar to a left-handed threefold $3 / 2$ helix was obtained for the modelled 8-mer according with the crystallographic structure. Coherently, this model showed HBs between the $\mathrm{N}-\mathrm{H}$ protons of the carbamate moieties at the 6-positions and the carbonyl oxygens at the 2-positions, with a distance of $2.634 \AA$ which is slightly shorter compared to the distance found in the X-ray structure. Then, cellulose tris(5-fluoro-2-methylphenylcarbamate) [92] and CDMPC [94] were constructed starting from left-handed threefold 3/ 2 helix of CTPC, by introducing the proper substitution on the phenyl rings.

In 2002, Okamoto and co-workers proposed a left-handed 4/3 helical structure as the most probable for ADMPC on the basis of 2D NOESY spectroscopy studies and computational modelling [70]. In this case, the initial structure of ADMPC was constructed by using the structure of amylose triisobutyrate postulated on the basis of X-ray analysis reported by Zugenmaier and Steinmeier [95], and a unit of ADMPC was obtained by changing the isobutyrate to 3,5-dimethylphenylcarbamate at the 2-, 3-, and 6-positions, and introducing $\mathrm{MeO}$ groups at the 1- and 4-positions. After minimization, this monomeric unit of ADMPC was used to construct a dimer, and the glycoside bond was parametrized using $-68.5^{\circ}$ and $-42.0^{\circ}$ as dihedral angles for $\phi$ and $\psi$ (Fig. 7), respectively. These values were determined by focused 2D NOESY experiments performed with low-molecular weight ADMPC (polymerization degree 100). This type of ADMPC could be studied by NMR due to its solubility in $\mathrm{CDCl}_{3}$, whereas high-molecular weight fragments are insoluble. Then, the optimized unit was used to construct the final 12-mer oligomer characterized by a left-handed four fold $4 / 3$ helix. Interestingly, the assigned structure was in accord with the results of Wenslow and Wang, who had identified a helical structure with less than six folds through structural analysis of ADMPC by solid-state NMR [96].

It is worth mentioning that in 1996, Wainer and Booth had generated a model for ADMPC starting from the conformational search of a glucoside dimer [97]. On this basis, the angles defining the glyco-

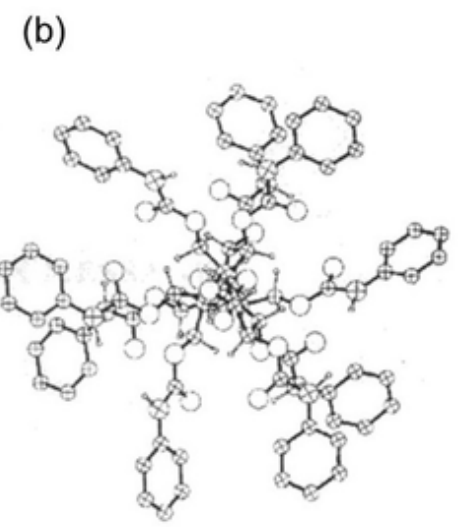

(d)

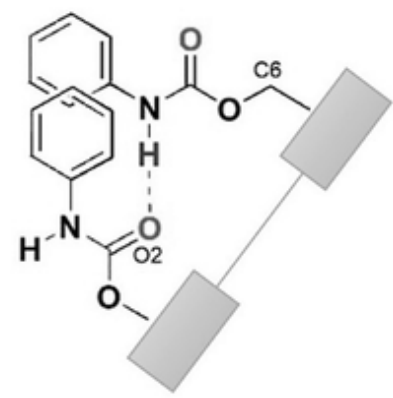

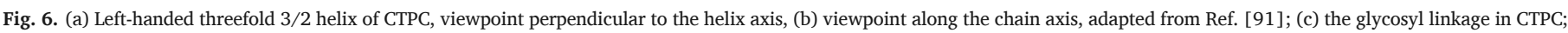
(d); drawing of intramolecular HB in CTPC. 


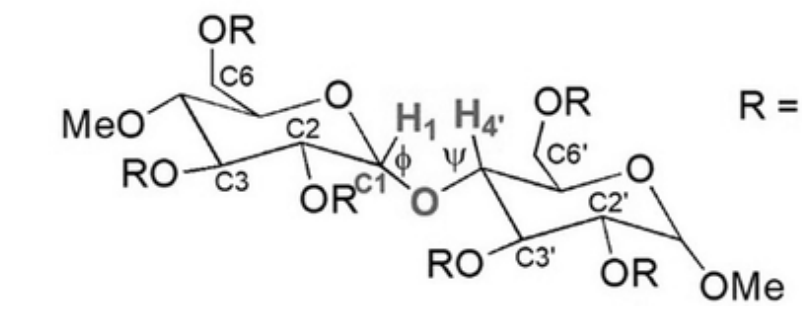

Fig. 7. Dimer structure of ADMPC. Two dihedral angles define the glycoside bond: $\phi\left(\mathrm{H}_{1}-\mathrm{C}_{1}-\mathrm{O}-\mathrm{C}_{4},\right)$ and $\psi\left(\mathrm{H}_{4},-\mathrm{C}_{4}-\mathrm{O}-\mathrm{C}_{1}\right)$.

side bond were found to be $\phi=-28^{\circ}$ and $\psi=-13.8^{\circ}$, and a polymer consisting of 20 monomeric units was built.

The modelled structures of CTPC, CDMPC and ADMPC (Fig. 8) present, as a common motif, a high-ordered structure held-up by intramolecular HBs between adjacent carbamate moieties. However, structural variations concerning both backbone and side chain affect the shape of the cavities available for the enantiomers. Indeed, the aromatic rings of CDMPC are arranged differently with respect to the phenyl ring of CTPC probably due the steric hindrance exerted by the methyl groups. Analogously, as the backbone changes from cellulose to amylose, the glycoside geometry changes, impacting helical type and cavities arrangement along the helical axis.

In 2007, Franses and co-workers studied the effects of the backbone and side chain on the chiral cavity of CDMPC and ADMPC by using attenuated total reflection infrared spectroscopy (ATR-IR), XRD, ${ }^{13} \mathrm{C}$ cross-polarization/magic-angle spinning (CP/MAS) and MAS solid-state NMR, and DFT modelling [98]. In this study, it was found that the strengths of the intramolecular HBs between $\mathrm{C}=\underline{\mathrm{O}}$ and $\mathrm{N}-\underline{\mathrm{H}}$ of the carbamate moiety of ADMPC and CDMPC are different. For CDMPC, cavities are slightly bigger than for ADMPC due to the differences in the polymer backbones, producing weaker intermolecular HBs in the cellulose-based polymer. The XRD results showed different packing arrangements for CDMPC and ADMPC in the solid state, resulting in different contacts of the adjacent polymer chains. For CDMPC, a larger distance between two adjacent chains compared to ADMPC was observed due to weaker intermolecular HBs. These differences in the packing arrangements of the polymer chains produce different nanostructures of the chiral cavities. Through DFT modelling a planar conformation was predicted for both ADMPC and CDMPC side chains.

In 1987, Steinmeier and Zugenmaier reported also for cellulose tribenzoate (CTB) a left-handed 3/2 helical structure [99]. Computa- tional models for CTB were reported by Francotte [100] and co-workers in 1991, and later, by Grinberg's group [101]. Cellulose trisbenzoate does not present as many HB interactions sites such as cellulose carbamates, this derivative lacking $\mathrm{N}-\underline{H}$ sites.

In 1990s, Okamoto and Chankvetadze studied by ${ }^{1} \mathrm{H}$ NMR and FT-IR the effects of structural variations of polysaccharide derivatives on intramolecular HBs between adjacent carbamate moieties [34-36]. Recently, these studies have been reviewed by Chankvetadze [16,17], therefore only representative results concerning cellulose tris(3,5-dichlorophenylcarbamate) (CDCPC), cellulose tris(3-chloro-5-methylphenylcarbamate) (CCMPC), and CDMPC are briefly reported herein in order to illustrate the concept (Fig. 9).

In Figure 9b, the ${ }^{1} \mathrm{H}$ NMR spectra of the $\mathrm{N}-\underline{H}$ region of the phenylcarbamate derivatives of cellulose are pictured for CDCPC, CCMPC, and CDMPC. The $\mathrm{N}-\underline{\mathrm{H}}$ proton chemical shifts of the derivatives were found dependent on the substituents on the aryl rings. The N-H resonance at the lowest field was assigned to the N-H proton at the 6-position $[34,36]$. By comparing the three spectra snapshots, it is observed that the chemical shifts of the N-H resonances shift downfield as the acidity of N-H increases. Recently, our group calculated at DFT level the $V$ maxima associated with the electropositive regions centred on $\mathrm{N}-\underline{H}$ hydrogens (Fig. 9a) [24], and $V_{\mathrm{S}, \max }$ values increased as the electron-withdrawing properties of the substituents on the aryl rings, in line with the NMR trend observed by Okamoto and Chankvetadze. Moreover, the authors confirmed the formation of intramolecular HBs between adjacent carbamate moieties in polysaccharide phenylcarbamates by IR spectroscopy focused in the $\mathrm{NH}$ region of the phenylcarbamates (Fig. 9c). The IR analyses indicated that electron-donating substituents on the phenyl ring (CDMPC) favoured intramolecular HB formation, whereas the presence of electron-withdrawing chlorines (CDCPC) on the phenyl ring was detrimental for intramolecular con-

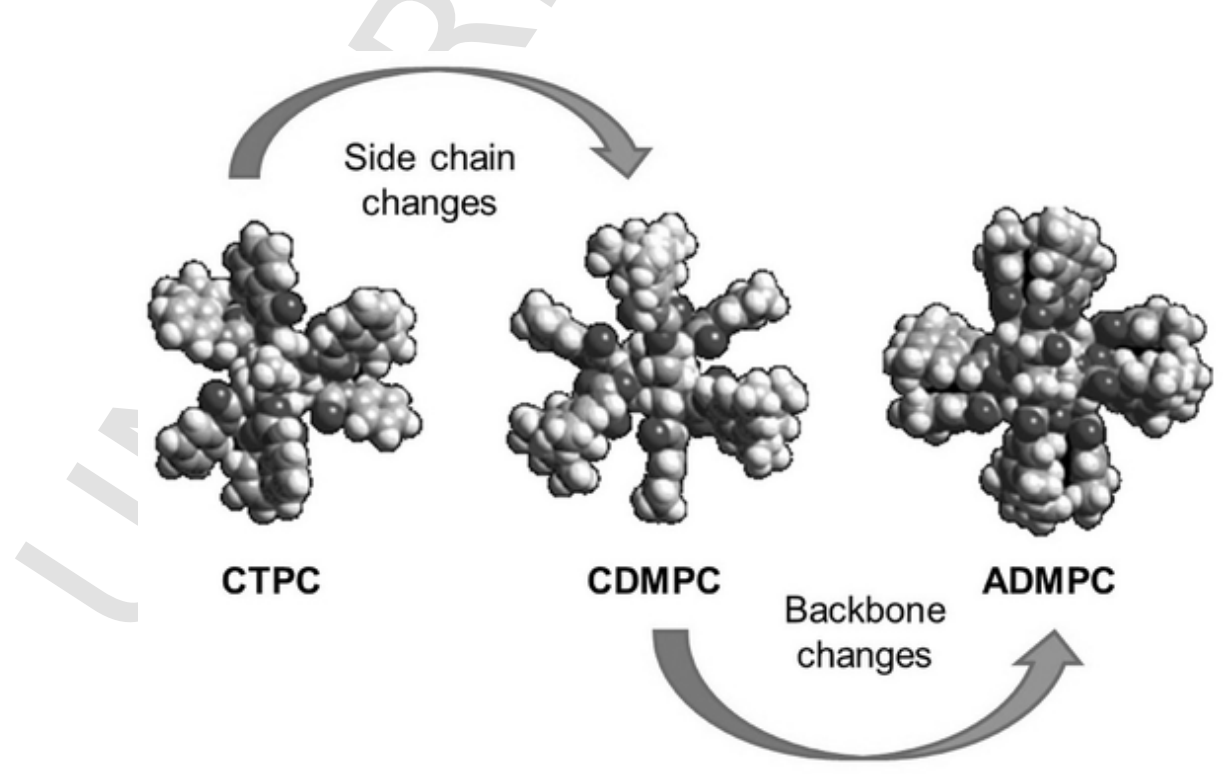

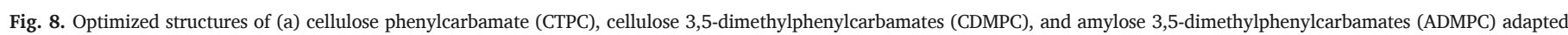
from Refs. [94,70]. Viewpoint is along the chiral axis. 

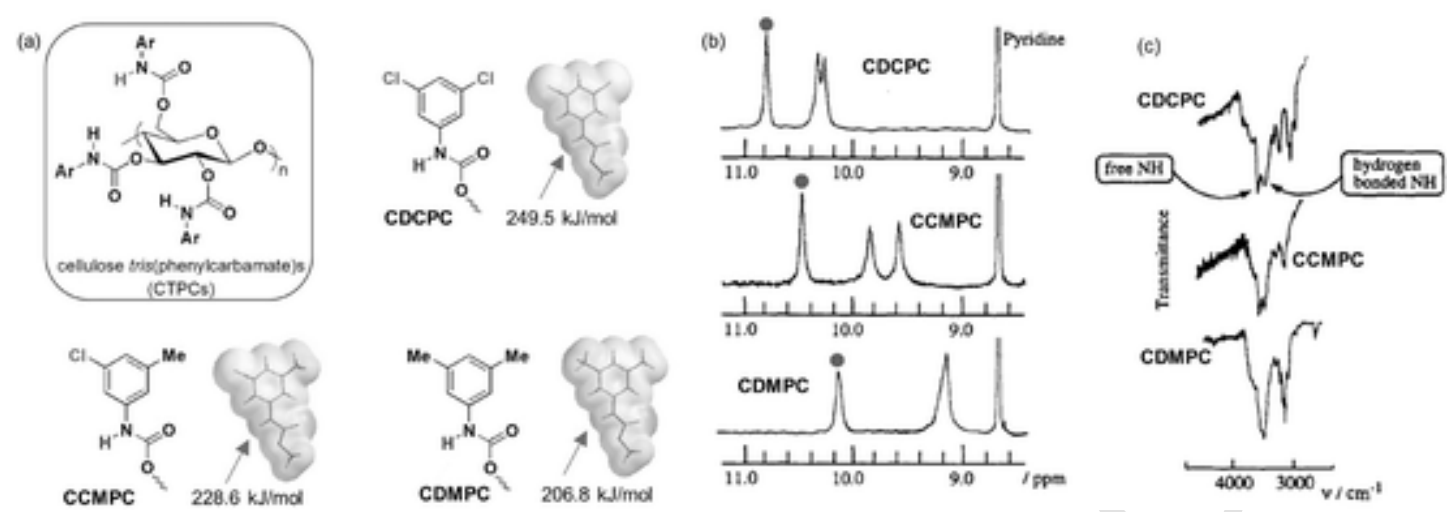

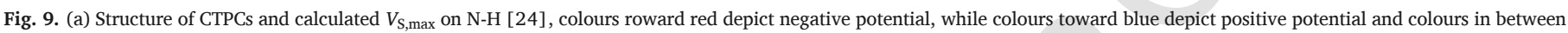

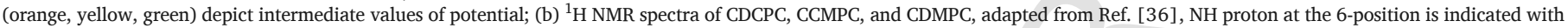
the red point; (c) IR spectra of CDCPC, CCMPC, and CDMPC, adapted from Ref. [36].

tacts. As shown in Figure 9c, FT-IR spectroscopy provided clear information about the ratio of the two types of situations involving the $\mathrm{N}-\mathrm{H}$ hydrogen of polysaccharide phenylcarbamates. Indeed, two diverse bands at different wavelengths around $3500 \mathrm{~cm}^{-1}$ are associated with the two molecular situations. Similar trends were observed for methyland chloro-substituted phenylcarbamates of amylose [35].

Different factors can affect intramolecular HBs and the high-ordered structure of polysaccharide benzoates and phenylcarbamate derivatives. The structure of cellulose benzoates and, consequently, their chiral recognition is more influenced by the conditions used for the preparation of the packing material than the corresponding phenylcarbamates. This difference is probably due to the different conformational stabilities between cellulose benzoates and phenylcarbamates [102]. Immobilization procedures can also alter the structure of polysaccharide derivatives because most of covalent immobilization technologies require under-derivatized polysaccharide in order to link it covalently to the silica surface. Recently, the topic has been comprehensively reviewed by Chankvetadze [18].

The effect of solvent on the high-ordered structure of polysaccharide derivatives has been investigated in some studies. In 2001, Wenslow and Wang reported ${ }^{1} \mathrm{H} /{ }^{13} \mathrm{C} \mathrm{CP} / \mathrm{MAS}$ solid-state NMR for monitoring the effects of several $n$-hexane mixtures containing ethanol, 2-propanol, and other alcohols as modifiers on the structure of commercial ADMPC-polymer-coated silica beads [96]. The CP/MAS spectrum of dry ADMPC displayed broad and amorphous-like carbon peaks representing backbone and side-chain carbons with no evidence of solvent incorporation. Treating the sorbent with $n$-hexane, solvent incorporation was clearly evident by solvent peaks present in the CP/MAS spectrum. The introduction of 2-propanol or ethanol, as alcohol modifiers in the $n$-hexane MP, produced the displacement of the incorporated $n$-hexane solvent accompanied by increased crystalline character of the polymer. In particular, 2-propanol proved to displace the incorporated $n$-hexane molecules more efficiently than ethanol. Similar results were obtained later by Franses and co-workers by exploring sorbent-solvent interactions for ADMPC with ATR-IR, XRD, CP/MAS and MAS NMR and DFT modelling $[71,103]$. The molecular modelling of complexes formed by solvent and a single modelled side chain allowed for predicting the IR wavenumbers, the HB interaction energies and the HB distances of the polymer side chains with $n$-hexane, methanol, ethanol, and 2-propanol. These results showed that the polymer rods form intra-rod HBs and contain many nanometer-sized cavities. IR results showed that the polymer has a wide distribution of intramolecular HBs. After absorption of a polar solvent, the structure of the polymer changed, whereas the absorption of $n$-hexane left the polymer essentially unchanged. The $\mathrm{OH}$ groups of the alcohols make strong $\mathrm{HB}$ complexes with the $\mathrm{C}=\underline{\mathrm{O}}$ and $\mathrm{N}-\underline{\mathrm{H}}$ groups of the polymer. Acetonitrile interacted with the polymer through dipole-dipole interactions, while $n$-hexane showed only dispersion interactions with the polymer. According with the results obtained by Wenslow, ${ }^{13} \mathrm{C} \mathrm{CP}$ /MAS solid-state NMR confirmed that the polar solvents change the distribution of the intramolecular HBs inside the polymer, producing changes in the polymer crystallinity and side-chain mobility. On this basis, the authors hypothesized that the polymer helical rods are re-organized upon absorption of alcohol or ACN, with the repeat distance between the helical rods upon such reorganization increasing as the solvent molecular size. This observation appears to be in accord with the 12-mer ADMPC structures obtained by Murad and co-workers through MD simulation performed by using pure methanol and the mixture $n$-heptane/2-propanol 90:10 as explicit solvents (Fig. 10) [104]. The configurations of ADMPC in different solvents had qualitatively the same left-handed helical nature, but their dimensions varied in different solvents. Representative structures of ADMPC in methanol (Fig. 10a) and n-heptane/2-propanol 90:10 (Fig. 10b) were generated by using clustering analysis from the last $20 \mathrm{~ns}$ of a $100 \mathrm{~ns}$ simulation of the system consisting of one ADMPC polymer and solvent molecules treated explicitly. Each molecule from top to bottom showed to have pitches with four monomers in each pitch in agreement with the 4/3 left-handed helical structure. Significantly, the average configuration of the 12-mer ADMPC in $n$-hep-
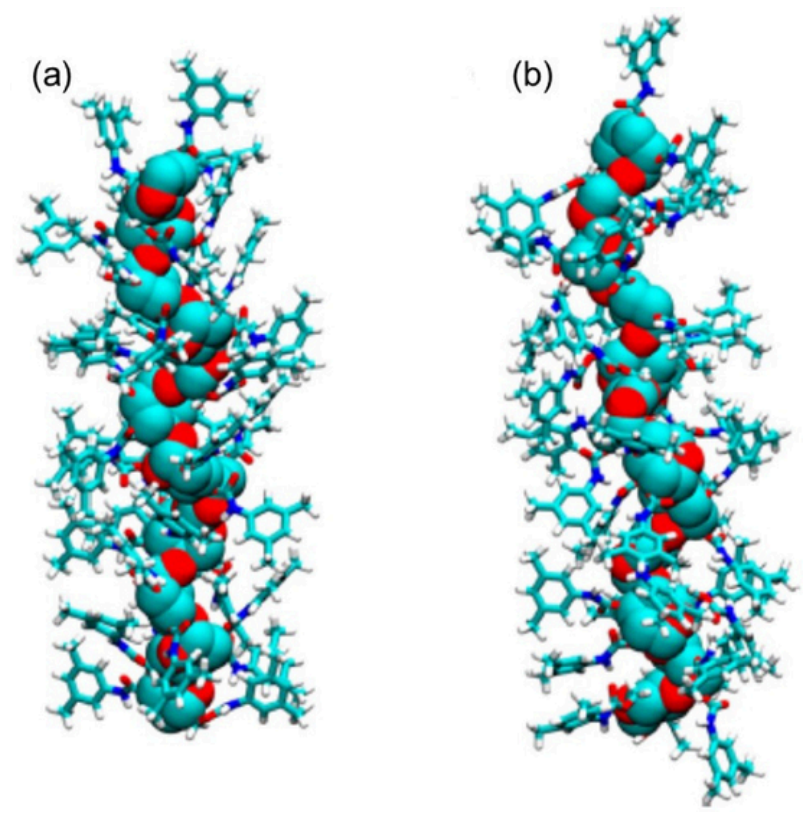

Fig. 10. Average structures of ADMPC in methanol (a) and $n$-heptane/2-propanol 90:10 (b), adapted from Ref. [104]: the backbone atoms are represented with vdW spheres and the derivatives are represented with sticks. Hydrogen atoms are in white, carbon atoms are in cyan, nitrogen atoms are in blue, and oxygen atoms are in red. 
tane/2-propanol 90:10 was longer (39.88 A) than in methanol (35.25 $\AA$ ), proving a solvent-dependent structural change of the polymer. Moreover, in accord with the results obtained by Wenslow and Wang, the 2-propanol molecules had a higher density at a closer distance to the backbone of ADMPC than methanol.

In 2008, Grinberg and co-workers reported the first study of ADMPC and CDMPC by vibrational circular dichroism (VCD) $[63,105]$. The comparison between experimental and theoretical VCD spectra showed that the two polymers undergo conformational changes as the polarity of the MP changes. In particular, spectra comparison evidenced the structural dfference between the two selectors. Indeed, an intramolecular $\mathrm{CH}_{3}-\pi$ contact was evidenced for the ADMPC, whereas the structure of CDMPC subunit showed all the phenyl side chain substituents far apart from each other.

Recently, Németh and Horváth observed isothermal hysteresis phenomena of retention and enantioselectivity on ADMPC using mixtures of 2-propanol and methanol [106]. On the basis of chromatographic results and van't Hoff analyses, the authors concluded that the only realistic mechanism to account for the hysteresis is a hindered transition of the high-ordered stucture of ADMPC. These results and the proposed hypothesis are in accord with previous results proving the strong tendency of polar solvents to be adsorbed on the polymer surface.

Temperature also affects chiral recognition pattern of polysaccharide derivatives. Several authors studied temperature-dependent effect on polysaccharide selectors, such as Ogura on cellulose triacetate [107], Marchal on cellulose tricarbamate [108], Grinberg on cellulose tris(4-methylbenzoate) [101], and Wang on ADMPC [109,110]. Very recently, Chankvetadze and co-workers noted opposite EEO under the same experimental conditions for the enantioseparation of ketoprofen on coated ADMPC before and after heating the column until $70^{\circ} \mathrm{C}$ [65]. The change in chiral recognition pattern could be due to a chemical or structural change in the chiral selector, chiral analyte, both of them or to enthalpy-entropy compensation. The authors hypothesized a temperature-dependent phase transition of the polymer which was confirmed by differential scanning calorimetry, observing transition start at temperatures as low as $30^{\circ} \mathrm{C}$.

\subsection{Intermolecular noncovalent interactions underlying enantiodiscrimination}

Enantiodiscrimination is the result of a multi-step process consisting of selectand adsorption (binding), diastereomeric complex formation (selection) and discrimination (function). On this basis, the polysaccharide selector discriminates the enantiomer pair by means of different noncovalent interaction set. Each single noncovalent interaction exhibits a stabilization power of the selector-enantiomer complex rather small. However, the sum of different interactions may cooperatively produce the energy gain which determines the enantiodiscrimination extent. In addition, interactions with the achiral support may also affect adsorption of the analyte. Moreover, as reported in the previous paragraphs, solvents have specific stereoelectronic properties and occupy a room inside cavities and ravines of the selectors, therefore MP may impact retention and selectivity by changing polymer structure, through solvation effect on analyte, and by stabilizing or destabilizing noncovalent interactions.

On the basis of the observations of Feibush [111], noncovalent interactions promoting enantiodiscrimination have sometimes been classified as a) leading (non-stereoselective) interactions which drive polymer-analyte association and determine the retention of a pair of enantiomers on a CSP, and b) secondary stereoselective interactions which determine enantiorecognition $[25,63]$. In this regard, it is worth pointing out that individual intermolecular interactions between the enantiomers and the CSP are achiral, whereas patterns consisting of multiple interactions may be stereoselective. In this perspective, depending on the 3D-constitution of each enantiomer, interactions may positively cooperate producing an extra-stabilization for the diastereoiso- meric complex which releases the second elute enantiomer. On the contrary, negative cooperation may occur between noncovalent interaction set producing an energetic destabilization of the other diastereoisomeric complex which releases the first eluted enantiomer. This concept makes it logic to use achiral descriptors to evaluate both retention and selectivity $[112,113]$.

The most popular model which explains chiral recognition in terms of noncovalent interaction cooperativity is the three-point interaction model proposed by Dalgliesh in 1952 [114]. Nevertheless, as evidenced by Del Rio and co-workers [112], the three-point interaction mechanism functions better for systems with few degrees of freedom and which do not present multiple binding modes with the CSPs.

On the other hand, in 1990s Wainer and co-workers proposed that chiral recognition on polysaccharide benzoates and phenylcarbamates is not due to 'point' interactions but to 'molecular interactions' through a conformationally-driven process [97]. On this basis, Wainer classified polysaccharide-based CSPs as Type II, "where the primary mechanism for the formation of the solute-CSP complex is through attractive interactions but where inclusion complexes also play an important role" [115]. Thus, the conformationally-driven mechanism consists of three steps: a) distribution of the solute to the stationary phase through HBs, b) conformational adjustments of the solute and insertion in the CSP, c) stabilization of the solute/CSP complex through electrostatic interactions [116].

Taking into account the peculiar role of the steric fit in chiral recognition events promoted on polysaccharide-based selector, descriptors such as globularity and flexibility were shown to impact retention and selectivity on both cellulose and amylose phenylcarbamates $[26,76]$.

Even if in the past computation approach was considered time-consuming and potentially unreliable with polymeric CSPs [75], nowadays molecular modelling has become more and more popular in order to explore enantiodiscrimination at microscopic level, and in the last decade non conventional enantioseparations have been also modelled contributing to increase quality and amount of molecular information theoretically accessible. As considered by Lipkowitz [117], modelling of enantioselection in chromatography involves the concept of potential energy surface, which determines the shape of the molecule, its dynamic features and interaction capability. In particular, for MD, the evolution of computational techniques and means allowed increased simulation times from picoseconds to nanoseconds. This is particularly important for modelling polysaccharide derivatives in order to reach stable selector-analyte equilibria over the simulation time. On the other hand, the intrinsic structural complexity of polysaccharide-based CSPs and the dependence of their recognition ability by their supramolecular structure are factors which make modelling enantiodiscrimination with these chiral selectors a challenging process $[23,44]$. In addition, the three-dimensional structure of polysaccharide-based CSPs is not well defined, thus this fact continues to make simulation of chiral chromatography setting involving these polymeric CSPs rather complicated.

In 1991, a pioneering study by Camilleri and co-workers modelled the enantioseparation of oxiracetam and related molecules on CTPC [118]. In the same year, Francotte and Wolf modelled CTB with the aim to address the structural features of the polymer and understand interaction mechanism, demonstrating that the sugar residues forming the polymer backbone participate in chiral discrimination [100].

In the years 1994-2002, Okamoto's group performed a series of studies on the chiral recognition of polysaccharide derivatives towards simple racemates (Fig. 11a) by using chromatography, NMR spectroscopy, and computational studies. These studies were described in detail by Lipkowitz [117], therefore they are briefly summarized herein. The tris(4-trimethylsilylphenylcarbamate) of cellulose, which is soluble in chloroform, was used to discriminate the enantiomer pair of trans-stilbene oxide $2[119,120]$ through NMR spectroscopy. In this case, the methine protons of 2 appeared splitted into singlets and the signal of (SS)-(-)-enantiomer was shifted downfield, confirming the chromatographic EEO (RR-SS) observed with the same selector un- 
(a)<smiles>c1ccc(C2CC2c2ccccc2)cc1</smiles>

3<smiles>Oc1ccc2ccccc2c1-c1c(O)ccc2ccccc12</smiles>

4<smiles>Cc1cccc(O)c1-c1c(C)cccc1O</smiles><smiles>O=C(c1ccccc1)C(O)c1ccccc1</smiles>

6<smiles>OC(c1c2ccccc2cc2ccccc12)C(F)(F)F</smiles>

(b)

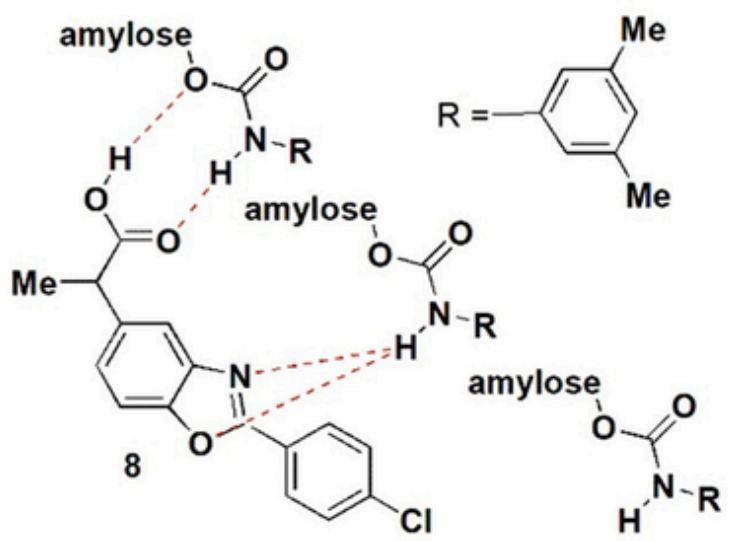

Fig. 11. (a) Structures of compounds 3-7; (b) proposed mechanism of binding showing HB sites for benoxaprofen (8).

der HPLC conditions. In 1995, the enantioseparation of 2 and the trans-1,2-diphenylcyclopropane (3) on CTPC were comparatively modelled through MD by using a 8-mer CSP [72]. HBs involving the oxirane ring and $\pi-\pi$ stacking involving aryl rings on both analyte and selector were found to be important noncovalent interactions. Later, the NMR investigation was extended to the tris(5-fluoro-2-methylphenylcarbamate) [92]. Focusing on compounds characterized by axial chirality, the authors showed that the fluorinated selector resolves enantiomers such as 1,1'-bi-2-naphthol (4) and 2,2'-dihydroxy-6,6'-dimethylbiphenyl (5) with high $\alpha>3$, and that, accordingly, large chemical shift differences between the signals of each enantiomer could be evidenced by ${ }^{1} \mathrm{H}$ and ${ }^{13} \mathrm{C}$ NMR. These findings were supported by MM energy minimization performed by using a 8-mer CSP. In 1999, the enantioseparation of benzoin (6) was modelled by using 9-mer of CDMPC and CTPC for MM energy minimization [94]. In 2002, the enantioseparation of 1-(9-anthryl)-2,2,2-trifluoroethanol (7) was studied on ADMPC [70]. HPLC analyses in $\mathrm{CHCl}_{3}$ performed on a chemically bonded CSP $(\mathrm{EEO}=R-S)$ and NMR experiments in $\mathrm{CDCl}_{3}$ demonstrated that $(S)-7$ is close to the $\mathrm{H} 1, \mathrm{H} 2$, and $\mathrm{H} 4$ protons of the glucose in the chiral groove of ADMPC through intermolecular HBs between the $\underline{\mathrm{OH}}$ proton of the analyte and probably the carbonyl oxygen of the carbamate group. In this regard, a modelled ADMPC was used for MM energy minimization of the (S)-7 /ADMPC complex, confirming the existence of a HB between the analyte and the carbonyl oxygen of the carbamate group at the 2-position of ADMPC. The distance between hydrogen and oxygen was found equal to $1.968 \AA$. Moreover, the anthryl ring was favourably posi- tioned close to the H1, H2, and H4 protons in accord with NMR and HPLC experiments.

These first studies demonstrated the pivotal role of the carbamate moieties of the polysaccharide derivatives in chiral recognition. The structural simplicity of the analytes studied in these first investigations (Fig. 11a) is probably due to the need of obtaining clear responses related to specific descriptors located on elemental frameworks. Over time advancements in spectroscopic techniques and computational methods allowed for studying more complex analyte structures.

In this regard, it is worth mentioning the study of Wainer and co-workers [97], where the enantioseparation of a more complex analyte was modelled by using a 20-mer ADMPC as the virtual selector. The authors docked the enantiomers of benoxaprofen (8) on ADMPC (Fig. 11b), assuming that the molecule is optimally docked when the maximum number of possible HBs is formed in combination to the lowest achievable interaction energy. The EEO of 8 being $R-S$, it was observed that when $(S)-\mathbf{8}$ was docked in the ravine in its lowest energy conformation, three simultaneous HBs were formed without significant molecular deformations. On the contrary, the docking of $(R)-8$ in the groove produced significant steric interactions between the solute and the stationary phase and the same HBs could not be obtained. Therefore, even though the two enantiomers form identical HBs, the two diastereomeric complexes differ in their stabilities leading to chiral discrimination. On this basis, the calculated $\alpha$ (1.52) was found to be consistent with the experimental selectivity (1.82). 
In 1997, Grinberg and co-workers modelled the enantioseparation of diol 9 and hydroxy ester 10 on a 6-mer of cellulose tris(4-methylphenylbenzoate) as built CSP [101] (Table 2). The interactive energy $\left(E_{\text {int }}\right)$ was determined by calculating the difference between the minimized energy of the docked molecules and the energy of the undocked molecules. Subtle changes in the structure of the two analytes indicated that $\pi-\pi$ interactions do not contribute to enantioselectivity. HB was observed to be a primary factor in the separation. Even though the chromatographic values do not agree with those obtained from MM energy minimizations, they showed the same trends (Table 2). Significantly, the authors considered that the disagreement in the values could depend on the fact that the MM calculations were performed by assuming gas phase conditions and with only a single short chain of cellulose. This comment highlighted the importance of solvent parametrization in modelling enantioseparation in order to account for solvent effects, and the need to use polymer frameworks of proper length in order to avoid the influence of the terminal groups of the framework.

In 2007, Wirth and co-workers [39] explored the enantiorecognition mechanism of $p$-O-tert-butyltyrosine allyl ester 11 on ADMPC by combining NMR experiments $\left({ }^{1} \mathrm{H}\right.$ and 2D NOESY) and MD simulations. At chromatographic level, 11 showed selectivity factor $\alpha=16$, with a strong binding of the L-enantiomer (EEO, D-L). In Figure 12, a snap-

Table 2

Chromatographic parameters, $\Delta \Delta G^{\circ}$ and calculated $\Delta E_{\text {int }}$ for enantiodiscrimination of 9 and 10 on cellulose tris(4-methylbenzoate) [101].

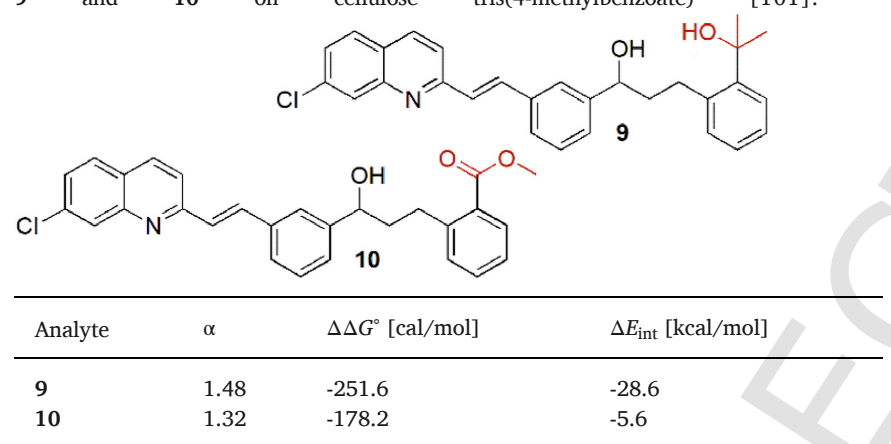

a $n$-hexane/ethanol 45:55

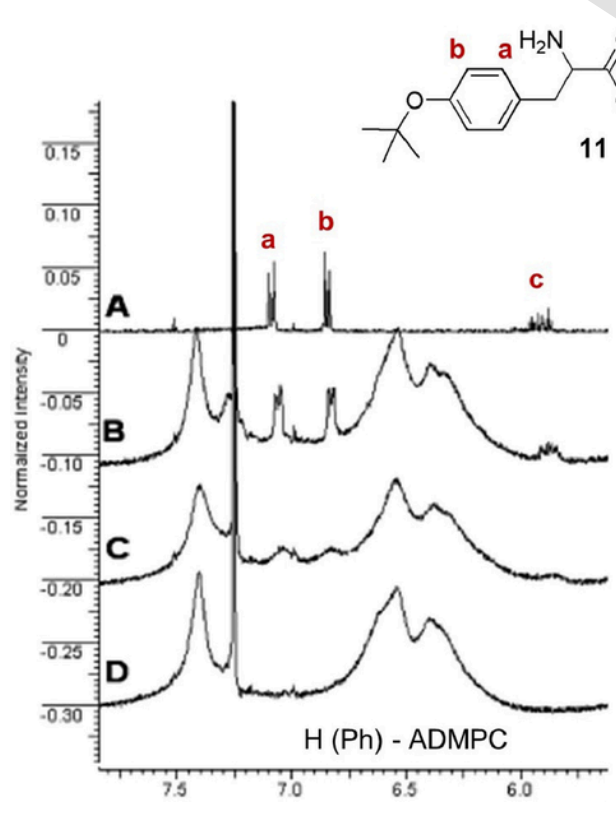

Fig. 12. ${ }^{1} \mathrm{H}$ NMR spectra variations of peaks a,b,c for the following samples (each with ethanesulfonic acid added) (adapted from Ref. [39]: A, O-tert-butyltyrosine (11) allyl ester alone; B, D-11 plus ADMPC; C, L-11 plus ADMPC; D, ADMPC alone. shot depicting ${ }^{1} \mathrm{H}$ NMR spectrum of peaks a,b,c of compound $\mathbf{1 1}$ alone in $\mathrm{CDCl}_{3}$, with the addition of ethanesulfonic acid, is reported (A), and the ${ }^{1} \mathrm{H}$ resonances of the analyte are narrow. Moreover, Figure $12 \mathrm{D}$ shows the spectrum for ADMPC in $\mathrm{CDCl}_{3}$ solution in the presence of ethanesulfonic acid. In Figures 12B and 12C the NMR spectra for the D- and L-enantiomers, respectively, in the presence of ADMPC and ethanesulfonic acid are reported. On this basis, it can be observed that in Figure $12 \mathrm{~B}$ the peaks a,b,c of compound $\mathbf{1 1}$ still show the spectral characteristics of the isolated molecule (A), therefore most D-enantiomers of the analyte seemed to be in the unbound state. On the contrary, for the L-enantiomer (C), the ${ }^{1} \mathrm{H}$ NMR resonances were greatly broadened reasonably due to a strong interaction with the polymeric material ADMPC. It is interesting to mention that in the absence of ethanesulfonic acid, no discernible broadening in the NMR spectrum of either enantiomer with ADMPC was observed, this result being consistent with chromatography behaviour, which showed that acid was necessary to achieve high enantioselectivity.

The 2D NOESY analysis confirmed the presence of a stronger complex between ADMPC and the L-enantiomer. In particular, it is worth mentioning that analyte/selector proton proximity found by $2 \mathrm{D}$ NMR analysis was used to address the initial placement of the enantiomer in the ADMPC complexes. For the MD simulation study, a 12-mer of ADMPC was used on the basis of the model reported by Okamoto's group [70]. A production run of 200 ps was performed, which generates 20 frames, each of which was subsequently minimized. The minimization process is useful to remove any unphysical geometries and finds the nearest minimum-energy structure. The polysaccharide backbone was fixed but the phenyl chains were allowed to move freely, and the solvent was treated implicitly by setting the dielectric constant to 4.8. From a close inspection of the energy-minimized structures of the selector/analyte complexes (Fig. 13), HB-driven interaction patterns were observed for both enantiomers. Indeed, HBs were found between the protonated amine group of $\mathbf{1 1}$ and the electronegative carbonyl oxygen of the ADMPC. In this study, the convention that a $\mathrm{HB}$ is formed when the distance between $\mathrm{H}$ and $\mathrm{O}$ is less than $3 \AA$ was adopted [39]. The amine group of the L-enantiomer showed a strong coordination with the three carbamate oxygens (Fig. 13c, interactions A', B', C' with distances of $2.010 \AA, 2.016 \AA$, and $2.883 \AA$, respectively). Such interactions are present for the D-enantiomer (Fig. 13b, A, B, C), but only B exhibited distance lower than $3 \AA$ (2.770 $⿱$ ). In addition, the L-enantiomer has a HB between its ether linkage and the $\mathrm{NH}$ group on the ADMPC carbamate side chain (D', $2.867 \AA$ ). It is worth mentioning that the authors argued that the strongest interactions are possible for the L-enantiomer because it is able to fit better into the ADMPC pocket (Fig. 13d) by folding over itself to maximize the contacts. On the contrary, the D-enantiomer (Fig. 13a) remained relatively linear because the energetic cost of maximizing the amine-carbonyl interaction is greater. The presence of the enantiomers perturbed the structure of the polymer differently. Even though this study still represents a seminal contribution in the field of chiral recognition processes promoted by polysaccharide-based CSPs, the used MD method presented some drawbacks such as limited production time (200 ps) and frame number (20), and the implicit treatment of solvent, probably related to limited computational power available at that time.In 2008, Franses and co-workers studied the impact of 13 structurally similar chiral solutes containing HB donors and acceptors, and aryl rings as elemental frameworks, on their HPLC retention and enantioresolution by using CDMPC as selector [121]. The HB between the functional groups of the solute and the $\mathrm{C}=\underline{\mathrm{O}}$ and $\mathrm{N}-\underline{\mathrm{H}}$ groups of the polymer were probed with ATR-IR for binary sorbent-solute systems. The CDMPC IR amide band wavenumbers changed significantly, indicating HBs of the polymer with the solutes. On the basis of HPLC data, IR data, and MD simulations performed by using a built 9-mer CDMPC, the interaction patterns were found to be consistent with the three-point attachment model, and support the hypothesis that significant enantioresolution requires at least three different synergistic interactions which can be 


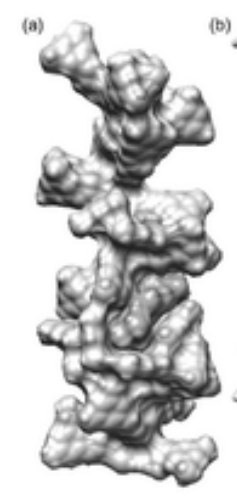

D-11/ADMPC complinx

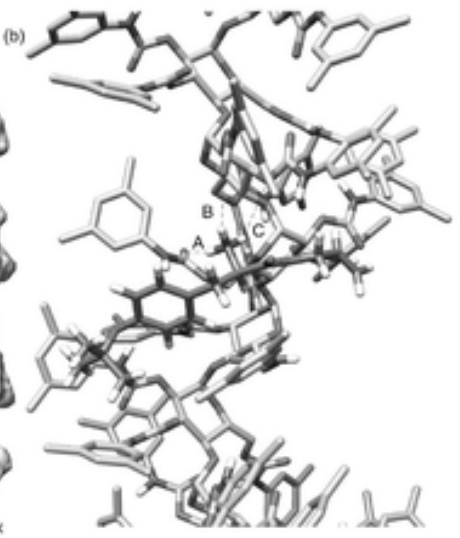

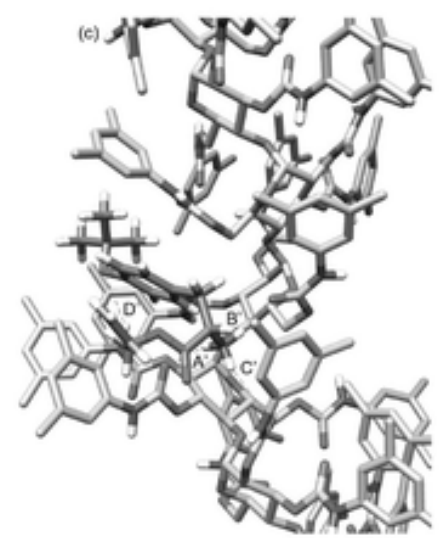

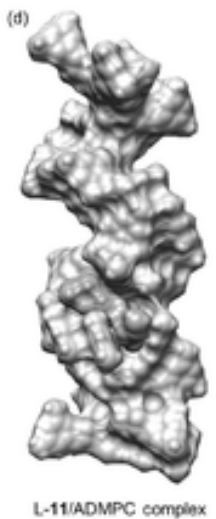

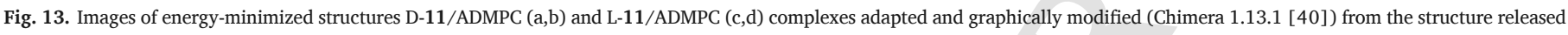
from Ref. [39]: (a,d) electron density surface view, (b,c) close-up view. HBs are indicated with red dotted lines.

a combination of steric hindrance, $\mathrm{HB}$, or $\pi-\pi$ interactions. The same combined approach was applied by the group to explore the key interactions of 2-amino-1-phenyl-1-propanol (12) with ADMPC and CDMPC [122]. It was hypothesized that the observed enantioselectivity in ADMPC was due to having three simultaneous interactions (two HBs and one $\pi-\pi)$ of the polymer with (-)-12 versus two interactions (one HB and one $\pi-\pi)$ with $(+)-\mathbf{1 2}$. It is worth mentioning author's observations about the fact that the MD-predicted enantioresolutions for the sorbent-solute systems (without solvent) were significantly higher than those observed in HPLC (with MP). One reason could be that in the MD simulations only the enthalpic contributions to the retention were considered, thus only a qualitative agreement may be expected because some factors such as solvent effects, entropic contributions, and the contributions from achiral sites of the CSP were underestimated.

In 2009, Grinberg and co-workers used a combined approach involving HPLC and VCD analyses, and DFT calculations to understand the mechanism underlying the enantioseparation of two aromatic $\alpha$-substituted alanine esters (13) on ADMPC and CDMPC [63]. In particular, by comparing VCD spectral differences of the two CSPs in the presence of the two analytes, detailed interactions involving different functional groups were identified, and the reversal of EEO on ADMPC associated with increasing solvent polarity was explained. Later, the same group applied this combined approach for studying of the enantioseparation of hexa-3,4-diene-3-ylbenzene (14) on CDMPC [123]. Van't Hoff plots for the retention factors showed that the retention of the two enantiomers is dominated by the enthalpy, while the plot for the selectivity is entropy driven. This suggested that the enantioselectivity is a result of inclusion of the enantiomers in the cavities of the selector. VCD spectra, along with DFT calculations supported the chromatographic findings.

In 2010, Zhou and co-workers modelled by MD simulation the chiral recognition of metalaxyl (15) and benalaxyl (16) on a 36-mer ADMPC in the presence of modified silica gel surface as the virtual CSP [124]. The chiral recognition was found to be mainly controlled by HBs. Nevertheless, the reliability of the final outcomes is affected by the absence of solvent, the simulation being performed in vacuum. Recently, Murad and co-workers proposed MD simulations of a series of enantioseparations using multiple ADMPC polymer strands (18-mer) coated on amorphous silica slab, treating the solvent explicitly [125]. Even though this study describes an interesting approach to design a model closer to a real system, the benefit of modelling the achiral support is not fully validated by the obtained results. Indeed, in some cases the modelled ADMPC/ silica slab setting produced similar results with respect to the modelled ADMPC alone [126].

Despite the pivotal role of HBs in chiral recognition promoted by polysaccharide derivates, hydrophobic, $\pi-\pi$ stacking, and vdW interactions also exhibited a prominent role in certain processes.
In 2012, Alcaro and co-workers explored the recognition mechanism of the enantioseparations of a series of pyrazole derivatives, demonstrating that analyte structural properties strongly impact the recognition pattern [127]. First, the enantioseparation mechanism of compounds 17 and 18 (Fig. 14a) on Chiralcel OJ-H (cellulose tris(4-methylbenzoate)) was studied by combining experimental analysis and molecular modelling. By using ethanol as MP, very high enantioselectivity was reported for both $17\left(k_{1}=4.6, k_{2}=336.0, \alpha=73.2\right)$ and $18\left(k_{1}=9.1\right.$, $k_{2}=723.7, \alpha=79.8$ ), with $R-S$ as EEO in both cases.

In this study, the polysaccharide-based selector was modelled starting from the left-handed threefold $3 / 2$ helix CTPC model described by Okamoto's group [72] and built on the basis of the structure obtained by Vogt and Zugenmaier by X-ray [91], replacing the carbamate by the benzoate moiety. A combined theoretical approach was used to explore the nature of selector-enantiomers association, which consisted of a) docking analysis by using a 4-mer oligomer as virtual selector (Fig. 14b, red units), and b) MD simulation (60 ns, ethanol as explicit solvent) by using a 16-mer oligomer (Fig. 14b, red 4-mer +12 yellow units). In order to prevent unrealistic distortions of the OJ-H CSP, the sugar ring atoms were restrained in their position by means of a constant force equal to $200 \mathrm{kcal} / \mathrm{mol}$. On the basis of this protocol, it was observed that the chiral recognition of $\mathbf{1 7}$ and $\mathbf{1 8}$ on Chiralcel OJ-H is driven by hydrophobic and $\pi-\pi$ stacking interactions rather than by HB contributions. Indeed, the thioamide moiety, the pyrazole nitrogen, and the ether oxygen were found to establish HB interactions prevalently with the solvent. Moreover, the difference in the free energy complexation calculated by docking accounted for experimental selectivity difference between $\mathbf{1 7}$ and $18, \Delta \Delta G_{\mathrm{S}-\mathrm{R}}$ was equal to -2.20 and $-2.60 \mathrm{kcal} / \mathrm{mol}$, respectively. Accordingly, the times of selector-enantiomer recognition extracted from MD trajectories were in agreement with the experimental EEO.

Later, Cirilli reported a study integrating chromatographic and thermodynamic van't Hoff analyses, and molecular modelling in order to explore recognition mechanism associated with the exceptional enantioseparation of pyrazole derivative $19(\alpha=207)$ with respect to the analogue compound $\mathbf{2 0}(\alpha=38)$ on a 4-methylbenzoate cellulose-based CSP under NP elution conditions ( $n$-hexane/2-propanol 30:70) (Fig. 15) [128]. In all cases $R$ - $S$ were determined as EEO. From the comparison of the experimental chromatographic data of 19 and of its $N$-methyl analogue $\mathbf{2 0}$ with those obtained by molecular modelling studies, it was found that the chiral resolving ability is modulated by $\mathrm{HB}$ interactions established between the thioamide moiety of the selectand and the carbonyl oxygen atoms of the selector. Each variation driven to weaken HBs caused a decrease in enantioselectivity, and lower selectivity factors were observed under PO conditions by using ethanol ( $\alpha=88$ (19), 22 (20)) or 2-propanol ( $\alpha=124$ (19), 24 (20)). This decrease in enantioselectivity passing from NP to PO modes 
(a)<smiles>CC(C)=CCOc1ccc(C2=NN(C(N)=S)C(c3ccccc3)C2)cc1</smiles>

(b)

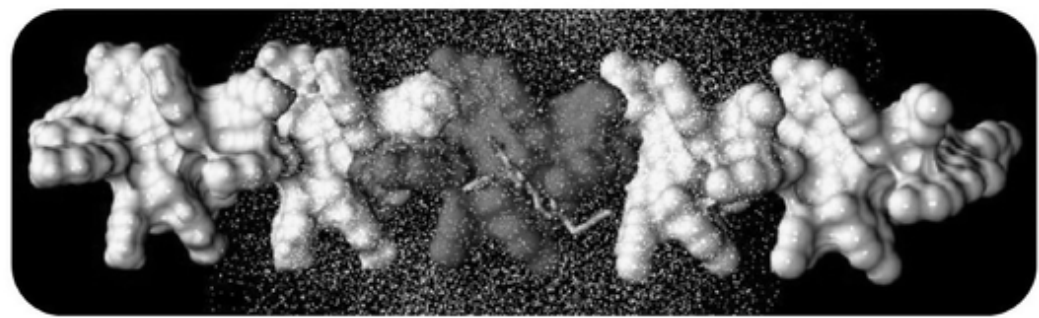

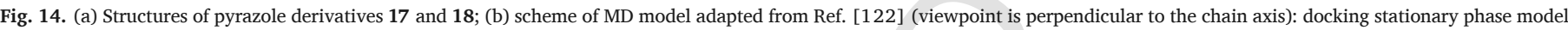
(4-mer) of cellulose tris(4-methylbenzoate) is coloured in red, units added in MD model (16-mer) are coloured in yellow, analyte is in green
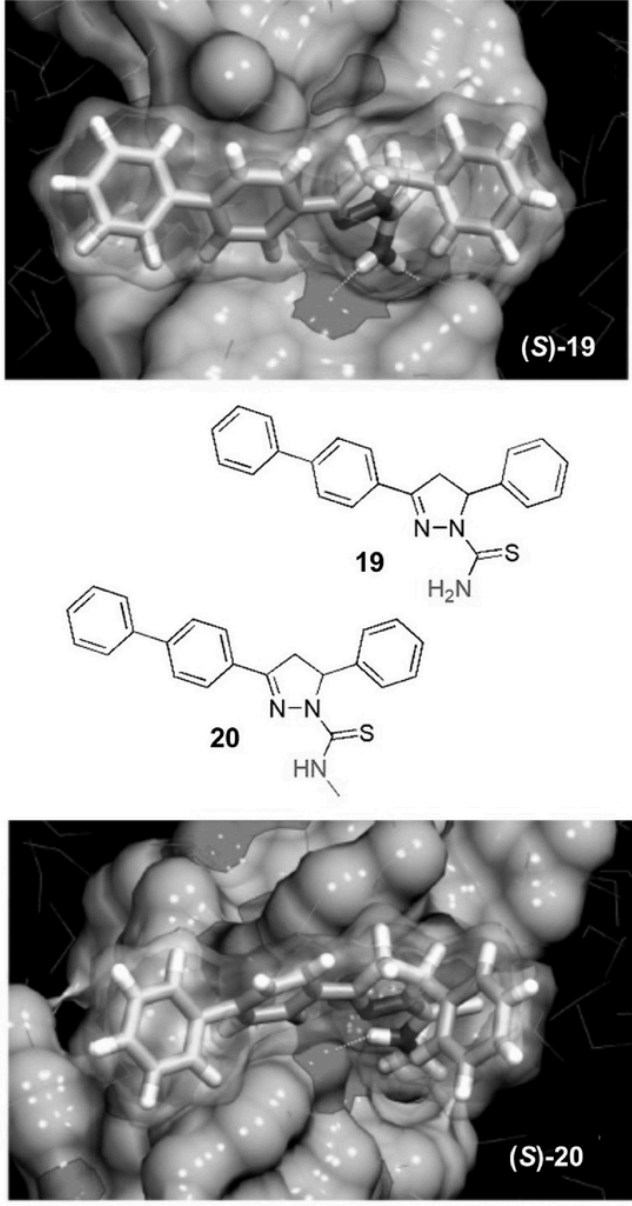

Fig. 15. Representative MD poses of (S)-19 and (S)-20 enantiomers interacting to the CSP, adapted from Ref. [128]. was also interpreted as a result of a lowered capability of the more retained enantiomer to establish strong HBs with complementary sites of the CSP. Indeed, taking into account representative MD poses reported in Fig. 15, all analytes localized the common biphenyl moiety between two 4-methylbenzoate side chains performing productive vdW and $\pi-\pi$ contacts, and HBs were observed between the $-\mathrm{NH}_{\mathrm{x}}$ thioamide group and the carbonyl oxygens. Nevertheless, due to the methyl thioamide, the $(S)$-enantiomer of $\mathbf{2 0}$ could show only one HB to CSP while $(S)-\mathbf{1 9}$ evidenced two HBs.

In general, selectivity factors higher than 5 determine long time analysis and large utilization of solvent, and are consequently not useful for applicative purposes. However, this kind of enantioseparations may be useful to gain information on enantiorecognition mechanism patterns which, acting at microscopic level, produce exceptional selectivity results at macroscopic level.

This type of behaviour was observed for the enantioseparation of 2-(benzylsulfinyl)benzamide (BSBA) $\mathbf{2 1}$ on cellulose tris(3,5-dichlorophenylcarbamate) (CDCPC) by Okamoto and co-workers [129,59]. It was observed that retention and selectivity factors of 21 on coated CDCPC increased in the order methanol < ethanol < 2-propanol, suggesting that HB is a dominant factor of enantiorecognition, particularly by using 2-propanol as MP $(\alpha=112)$ (Fig. 16a).

In the last few years, enantioseparation of BSBA and other sulfoxides has been intensively investigated. In 2017, West and co-workers studied the enantioseparation of BSBA and other chiral sulfoxides on cellulose-based selectors under supercritical fluid chromatography (SFC) conditions [130]. Molecular modelling studies indicated that BSBA could adopt a folded U-shaped conformation (Fig. 16b), which was efficiently discriminated compared to other conformations, hypothesizing the occurrence of a conformationally-driven enantiorecognition process. In other words, not only the type of interactions occurring between analyte and selector, but also the steric fit of the analyte in the chiral groove impact the enantiorecognition process. Later, Chankvetadze and co-workers explored the enantioseparation of 2-(sulfinyl)benzoyl derivatives either via variations of MP or via structural variations of analytes and cellulose-based selectors [131-133]. From this study, the peculiar enantioseparation behaviour of BSBA appears to be driven by a HB between the $\mathrm{NH}_{2}$ moiety of the amide group of the analyte and the carbonyl oxygen of the selector. In fact, as the amide moiety was replaced with methoxy or hydroxyl groups, the corresponding ester 
(a)

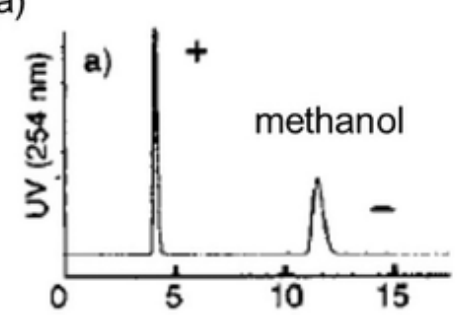<smiles>NC(=O)c1ccccc1S(=O)Cc1ccccc1</smiles>

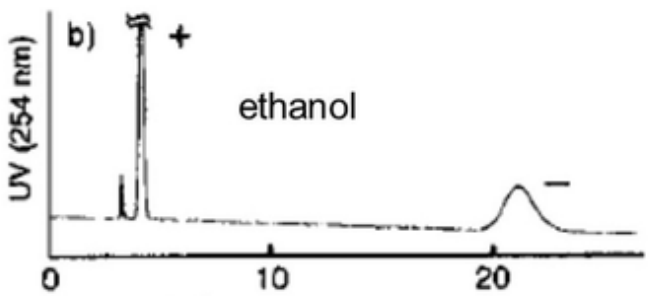

21

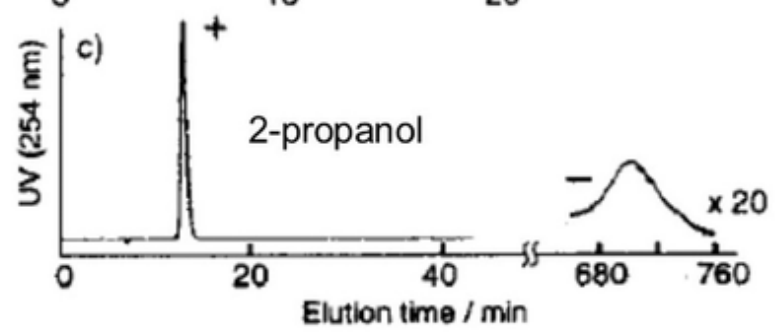

(b)

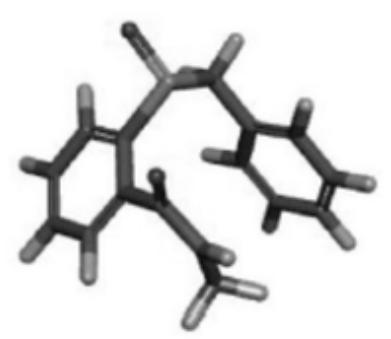

(c)

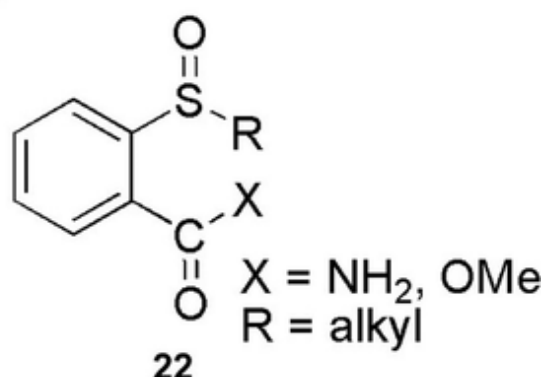

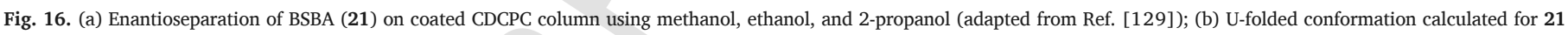
by MMFF (adapted from Ref. [130]); (c) structure of 2-(sulfinyl)benzoyl derivative 22 .

and acid derivatives were less enantioseparated than BSBA. Analogously, the same effect was observed by introducing a methyl group on the $\mathrm{N}$ atom. Very recently, Cirilli and co-workers reported the results of a chromatographic study aiming to evaluate the impact of subtle structural variations of the analyte on the enantioseparation of amides and esters containing the 2-(sulfinyl)benzoyl framework (22) (Fig. 16c) on the immobilized CDCPC [134]. By using $n$-hexane-based mixture and 2-propanol as MPs, the best degree of enantioseparation was achieved for the amide derivative bearing a $n$-pentyl chain. These results demonstrated that hydrophobic forces can lead to a modulation of the main noncovalent interaction driving enantiorecognition. Consequently, dispersive forces between the aryl group of the selector and the alkyl chain of chiral sulfoxide, and repulsive forces of steric nature can also act in positive or negative cooperativity with the main $\mathrm{HB}$ exerted by the $\mathrm{NH}_{2}$ group.

The composition of the MP can have a very important effect on chiral recognition, therefore the effect of solvent should be considered in theoretical computational studies.

Recently, explicit-solvent MD simulations were performed by Murad and co-workers in order to elucidate the chiral recognition mechanism of the enantioseparation of flavanone (23) enantiomers on ADMPC
[104]. For this purpose, a 12-mer oligomer was built on the basis of the parametrization reported by Okamoto's group, and methanol or heptane/IPA (90/10) were used as explicit solvents. It was found that the number of solvent molecules around each enantiomer of flavanone correlates well with the interaction between the ADMPC and the enantiomer in terms of electrostatic interaction energy. Indeed, the number of solvent molecules within $5 \AA$ of the drug molecule significantly decreases when the drug molecule interacts with the ADMPC polymer. In the absence of the ADMPC, the enantiomers form HBs with methanol or 2-propanol, or hydrophobic interactions with hexane, generating a stable first solvation shell. MD analysis reveals that the lifetime of the HBs formed between the flavanone enantiomer and ADMPC polymers correlates well with both selectivity of the enantiomers ( 0.9 and 0.4 in heptane/2-propanol and methanol, respectively) and EEO $(R-S)$, the $S$-flavanone proving to have a longer HB lifetime with ADMPC than the enantiomer $R . \pi-\pi$ Interactions did not impact separation factor, whereas steric effects involving the planar rings of the flavanone and the chiral selector clearly did play an important role in tuning the efficacy of selector-analyte HBs which are the main drivers of the enantioseparation. 
In a different situation, recently, the same authors observed that $\pi-\pi$ interaction can positively cooperate with HB formation [126]. A 12-mer ADMPC, weakly restrained, was used to model the enantiosepation of 6 on ADMPC ( $\mathrm{EEO}_{\text {experimental }}=R-S$ ) by using heptane/2-propanol as explicit solvent, with $100 \mathrm{~ns}$ as MD production time (Fig. 17).

It was found that the $\mathrm{C}=\underline{\mathrm{O}}$ acceptor of benzoin forms the longest-lived HBs with the N- $\underline{H}$ donor of ADMPC more for the $S$-enantiomer compared to the $R$-enantiomer. Then, HBs between the $\underline{\mathrm{O}}-\mathrm{H}$ acceptor of benzoin and the N- $\underline{\mathrm{H}}$ donor of ADMPC were also observed with slightly favourable lifetimes for $R-6$. The $\mathrm{OH}$ donor of benzoin with the $\mathrm{C}=\mathrm{O}$ acceptor of ADMPC form the third most significant $\mathrm{HB}$, with a lifetime higher for the $S$-enantiomer compared to $R$-enantiomer. Moreover, formation of the HBs between the enantiomers and the ADMPC was found to be assisted by $\pi-\pi$ interactions which stabilize sterically and electronically HB formation.

In 2016, Huang and co-workers modelled the enantioselection of chiral pyrazole derivative 24 on cellulose tris(4-methylbenzoate) by means of MD (100 ps of simulation time) accounting for solvent implicitly [80]. In this study, a 12-mer fragment was built to model cellulose tris(4-methylbenzoate), with the terminal monomers replaced by methyl groups, and seven mixtures were used as custom solvents.

DC values were set by the authors to represent the experimental conditions as follows: $n$-hexane/ethanol $(70 / 30)(\mathrm{DC}=9.06), n$-hexane/ 2- propanol $(60 / 40)(D C=8.58)$, pure ethanol $(D C=25.80)$ and pure 2-propanol ( $\mathrm{DC}=18.62$ ). In addition, three reference solvent conditions, vacuum $(\mathrm{DC}=1)$, pure $n$-hexane $(\mathrm{DC}=1.89)$ and water ( $D C=81.00$ ) were also considered in order to explore the solvent effect systematically. In polar solvents the $S$-enantiomer/CSP complex appeared more stable than the $R$-enantiomer/CSP complex, according with the experimental EEO reported for $\mathbf{2 4}$ by using PO solvents or NP with an alcohol content $>30 \%$ [135]. The sequence $S$ - $R$ under PO conditions was explained with a mechanism driven by nonpolar interactions (Fig. 18a,b). In particular, using 2-propanol as explicit solvent, the $(R)$-enantiomer is inset in the groove (a), and 5-(2-chlorophenyl) is bare not involved in specific interactions. On the contrary, the (S)-enantiomer is inset in the groove (b) and surrounded by the side chains of the selector, with a $\pi$ - $\pi$ interaction formed between the 5-phenyl of the enantiomer and the benzene ring of the polymer. These observations were also confirmed by calculated $E_{\mathrm{vdW}}$ energies lower than the $E_{\mathrm{es}}$ contribution to interactions energy, with the interaction energy of the $R$-enantiomer/CSP complex higher than the energy of $S$-enantiomer/CSP complex. On the contrary, calculations predicted inversion of EEO $(S-R)$ in non polar medium and in the vacuum (Fig. $18 \mathrm{c}$,d) where HBs could occur between the hydroxyl proton of $\mathbf{2 4}$ and the carbonyl oxygen of the CSP. Nevertheless, experimental confirmation of the predicted EEO in non polar medium was not reported affecting the reliability of the reported conclusions.

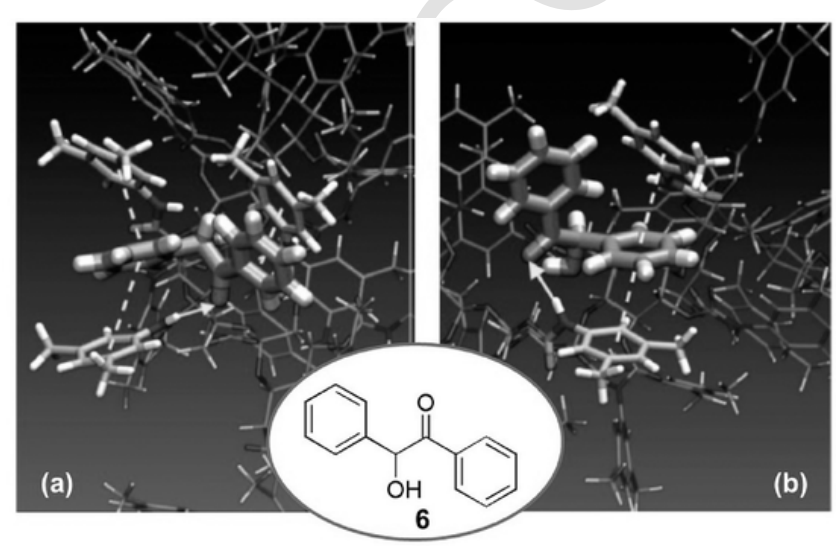

Fig. 17. MD simulation of enantiodistinction contact between benxoin 6 and ADMPC: (a) $S$-6/ADMPC complex, (b) R-6/ADMPC complexes. Adapted from ref. [126].

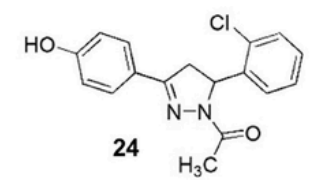

(a)

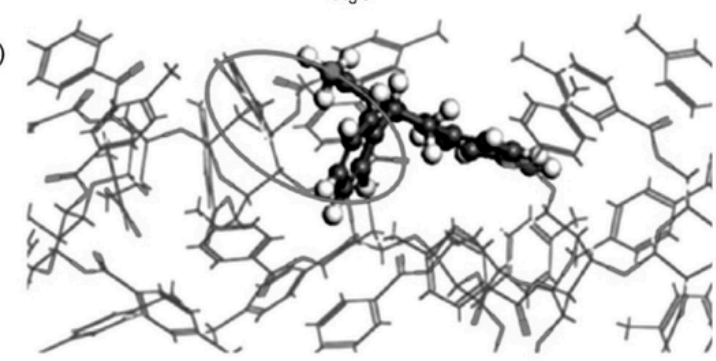

(b)

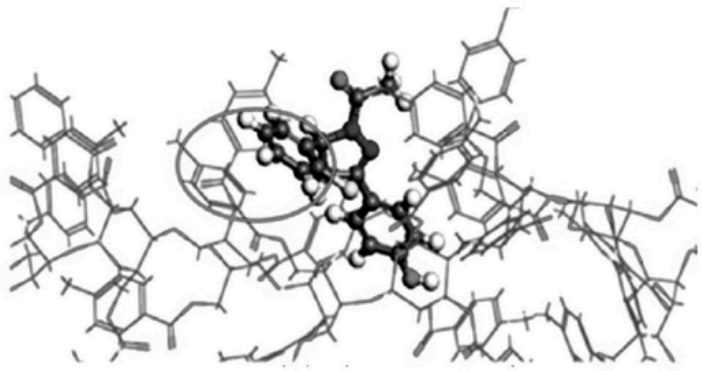

(c)

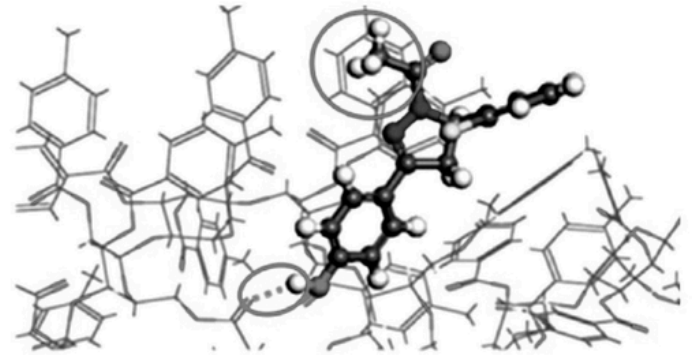

(d)

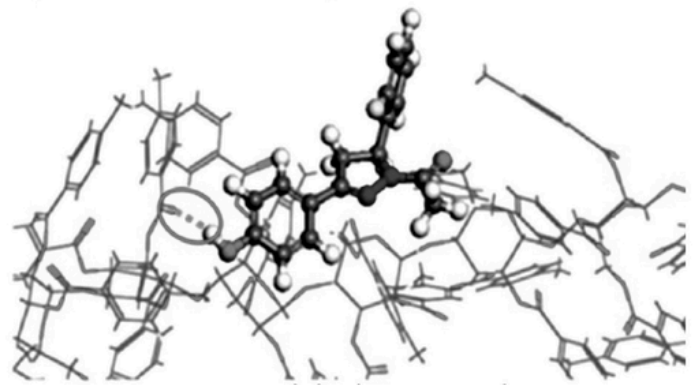

Fig. 18. Mean energy conformations of the complexes between enantiomers of pyrazole 24 and cellulose tris(4-methylbenzoate) (12-mer): (a) (R)-24/selector, 2-propanol (DC = 18.62), (b) $(S)$-24/selector, 2-propanol (DC = 18.62), (c) $(R)-24 /$ selector, vacuum $(\mathrm{DC}=1.00)$, (d) $(S)-24 /$ selector, vacuum $(\mathrm{DC}=1.00)$. Adapted from ref. [80].

As noted on the basis of the cases discussed above, hydrophobic and vdW interactions, which are the weakest noncovalent interactions, can also impact enantiorecognition by determining hydrophobic contacts into the grooves and ravines of polysaccharide-based selectors. In this regard, Kim and co-workers demonstrated by MD simulations that retention of a series of $N$-phthaloyl and $N$-naphthaloyl $\alpha$-amino acids on cellulose- and amylose-based CSPs, under NP elution conditions, is also controlled by vdW interactions [79]. These interactions, in some cases, proved to be dominant compared to electrostatic interactions such as HB. For the purpose, the interaction configurations of 10000 pairs of built cellulose (6-mer) and amylose (8-mer) oligomers and enantiomers were investigated by MD and, among all pairs, 100 low-energy pair configurations were selected, and average interaction energies $\left(E_{\text {tot }}\right)$ including $E_{\mathrm{vdW}}$ and $E_{\mathrm{es}}$ were calculated. 
As representative examples, compounds 25 and 26 (Fig. 19) are considered herein, and in Table 3 chromatographic parameters for their enantioseparation on Chiralcel OF (containing cellulose tris(4-chlorophenylcarbamate)) and Chiralpak AD (containing ADMPC), and related MD energies are reported. Retention of compound 25 on Chiralcel OF and compound $\mathbf{2 6}$ on Chiralpak AD were shown to be controlled by electrostatic and vdW interactions, respectively.

At the molecular level, for compounds 25 and 26 (Fig. 19), $V$ analysis revealed the presence of regions with high (red) and low (blue) electron density. Moreover, large hydrophobic regions (green) were present in both structures, which govern the occurrence of electrostatic or vdW interactions depending on the location of the analyte into the groove. On this basis, MD allowed to rationalize the enantioseparation results, showing that compound $\mathbf{2 5}$ locates the polar carboxyl moiety toward the groove of the cellulose selector, whereas compound $\mathbf{2 6}$ is oriented in the ADMPC groove pointing the hydrophobic regions toward the groove. By using MM calculations, the same group found that steric interactions exert a cooperative effect tuning chiral recognition mechanisms driven by other interactions such as HBs [136,137]. In a first study, pyrethroic acid esters 27 (Fig. 20a) were well resolved on Chiralcel OD and OF as chiral columns, under NP elution conditions. MM was used to calculate the interaction energy of the lowest energy conformations between 3-mer units of selectors and each enantiomer analyte. Considering the enantioseparation of cis-pyrethroic acid methylesters on Chiralcel OD (Fig. 20b), the lowest energy conformations of both CSP/enantiomer complexes showed two HBs occurring between analyte and selector. In particular, the alkenyl substituent R points toward the CSP in the cis form, originating a steric hindrance with the methyl group on the CSP. The sequence $S-R$ as experimental EEO could be justified by the decreasing binding ability between CSP and the (S)-enantiomer due to the higher steric hindrance between $\mathrm{R}$ and the methyl group of the CSP.

Analogously, for the enantioseparation of the fungicide ethaboxam 28 (Fig. 20c) on amylose and cellulose-based CSPs, the interaction en-

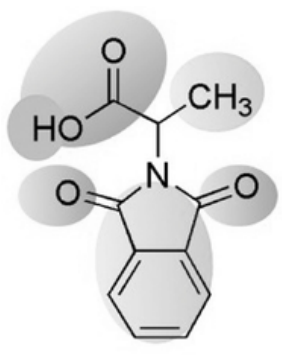

25

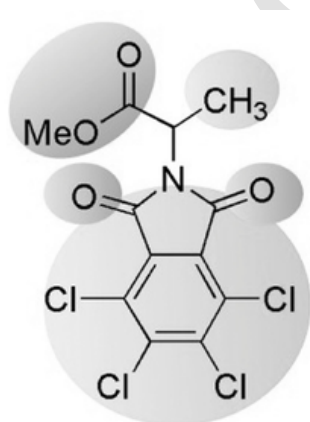

26
Fig. 19. Qualitative representation of regions of high electron density (red) and low electron density (blue) derived from $V_{S}$ analysis of compounds 25 and 26 [79]. Hydrophobic regions are reported in green. ergies between the enantiomers and the polysaccharide-derivatives modelled as 7-mer oligomers were calculated by MM [137]. The EEO derived from the interaction energies were $S$ - $R$ for cellulose-based CSP and $R$-S for the amylose-based selector, evidencing a EEO reversal dependent on the backbone nature. In this case, the steric repulsion between the thiophene group and the alkyl group of the CSP affects the efficacy of the HBs as the heteroaromatic group pointed to the groove.

In 2014, our group reported for the first time that a XB $[49,138]$ can drive HPLC enantioseparation of iodinated 4,4'-bipyridine analyte 29 (XB donor) (Fig. 21a) by using CDMPC as the chiral selector (XB acceptor), broadening the range of interactions which are active in HPLC environment [24]. The efficient enantioseparation resulted from the interaction of the electropositive $\sigma$-hole regions centred on iodine with the carbonyl groups of the carbamate cellulose backbone of the selector (Fig. 21b) $[24,49]$.

Later, with the aim of extending the concept of XB-driven HPLC enantioseparations, we have evaluated the performance of cellulose-based CDMPC and amylose-based columns in the enantioseparation of a large set of chiral 4,4'-bipyridines 30 bearing 3 to 6 halogens in various positions (Fig. 21c) [27,139]. In these compounds, halogens serve as $\sigma$-hole sites and as inductors of chirality by restricted rotation around the 4,4'-bipyridyl bond [140]. Examination of the collected chromatographic data showed that the recognition mechanism depends on substitution pattern, steric fit and chromatographic system (polysaccharide-based CSP and MP). Whereas the $\sigma$-holes on halogens were shown to exclusively control enantioseparation on CDMPC, a mixed enantioseparation mechanism controlled by halogen-dependent interactions and analyte steric fit seemed to be active on ADMPC. In this last case, the chromatographic outcomes depended on both volume and XBD ability of the analyte [139].

Interestingly, for both cellulose- and amylose-based CSP, a dramatic drop of selectivity was observed by using the chloro-substituted CCMPC and ACMPC as chiral columns due to the reduced XBA ability of the carbonyl. Moreover, the polar interaction contribution to the selectivity was progressively suppressed by changing the MP from NP to EtOH or $\mathrm{MeOH}$ because alcohols exerted a cap-effect on the carbonyls of the CSP by means of competitive HB interactions (Fig. 22).

Since $\sigma$-holes are regions of electronic charge density depletion, computationally calculated $V_{S}$ has been fruitfully used as an indicator of the anisotropy of the molecular charge distribution. Indeed, the capability of a halogen to behave as $\sigma$-hole donor could be estimated by the $V_{\mathrm{S} \text {,max }}$ calculated on its surface. On the other side, the $V_{\mathrm{s}, \min }$ value of the CSP carbonyl oxygens is also a good indicator of its performance for the separation of $\sigma$-hole based substrates. Indeed, for CDMPC or ADMPC containing the 3,5-dimethylphenyl framework, a $V_{\mathrm{s}, \min }$ value around $-170 \mathrm{~kJ} /$ mol was calculated on the carbonyl oxygens, which is similar to other typical acceptors like acetone $(-177 \mathrm{~kJ} / \mathrm{mol})$. These results supported theoretically the experimental behaviour of CDMPC and ADMPC as good $\sigma$-hole acceptor. On the contrary, the higher $V_{\mathrm{s}, \min }$ values (from -155 to $-160 \mathrm{~kJ} / \mathrm{mol}$ ) for the carbonyls of CSPs bearing chlorines such as CCMPC and ACMPC indicated a reduced capability as good $\sigma$-hole acceptor.

Table 3

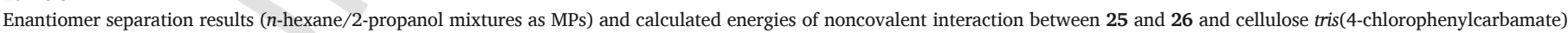
andADMPC, respectively [79].

\begin{tabular}{|c|c|c|c|c|c|c|c|c|c|}
\hline Compound & Column & $k_{1}^{a}$ & $\alpha^{b}$ & $R_{\mathrm{S}}{ }^{c}$ & $\mathrm{EEO}_{\text {exp }}$ & $E_{\mathrm{es}}{ }^{d}$ & $E_{\mathrm{vdW}}{ }^{d}$ & $E_{\text {tot }}{ }^{d}$ & $\mathrm{EEO}_{\text {calc }}$ \\
\hline (D)-25 & Chiralcel OF & 15.41 & 1.50 & 3.93 & D-L & -34.938 & -5.178 & -40.116 & D-L \\
\hline (L)-25 & & & & & & -38.215 & -8.334 & -46.549 & \\
\hline (L)-26 & Chiralpak AD & 2.70 & 1.77 & 5.28 & L-D & -3.423 & -25.402 & -28.825 & L-D \\
\hline (D)-26 & & & & & & -6.726 & -25.210 & -31.941 & \\
\hline
\end{tabular}

\footnotetext{
a retention factor of the first eluted enantiomer

b selectivity factor

c resolution factor

${ }^{\mathrm{d}} E_{\text {interaction }}=E_{\text {electrostatic }}+E_{\mathrm{van} \text { der Waals }}\left(E_{\mathrm{tot}}=E_{\mathrm{es}}+E_{\mathrm{vdW}}\right)$
} 


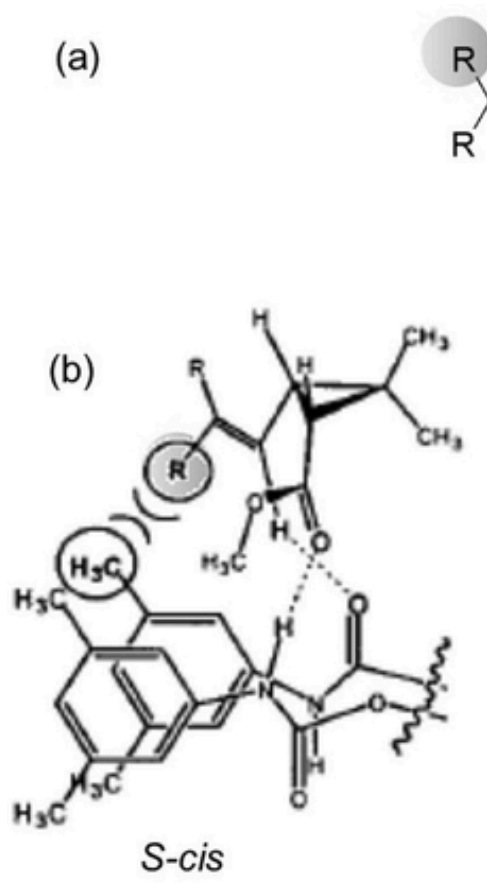<smiles>[R]OC(=O)C1C(C=C([R])[R])C1(C)C</smiles>

27

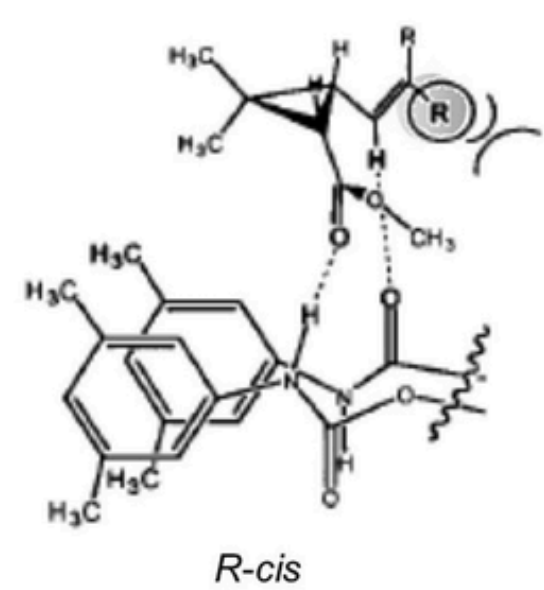

(c)

\section{Ethaboxam (28)}

Fig. 20. (a) Structure of pyretroic acid esters; (b) steric effect of group R in the enantioseparation of cis-27 on Chiralcel OD. Adapted from Ref. [136]; (b) structure of ethaboxam (28).

The interaction modes of eight 4,4'-bipyridine pairs with CDMPC and ADMPC were simulated by MD calculations [73]. $35 \mathrm{X} \cdots \mathrm{O}$ and 10 $\mathrm{X} \bullet \bullet \pi$ contacts were found, with a clear prevalence of $\mathrm{I} \bullet \bullet \mathrm{O}$ contacts (32). In Figure 23, the results of $10 \mathrm{~ns} \mathrm{MD}$ production are reported for the enantioseparation of compound 30a in terms of occupancy graphs evidencing the room occupied by each enantiomer during the production time. Moreover, the involvement of the carbonyl site was statistically evaluated and the highest values were observed for the carbonyl at 3and 6-positions. Interestingly, the number of interactions observed for iodinated, brominated and chlorinated compounds $\mathbf{3 0}$ tended to reflect the order $\mathrm{I}>\mathrm{Br}>\mathrm{Cl}$, in agreement with $\sigma$-hole depth trend and experimental outcomes.

Recently, the same strategy has been applied to fluorinated sulfur-containing 4,4'-bipyridines with the aim to detect and study chalcogen bond (ChB) in solution [141,142]. Indeed, these 4,4'-bipyridines contain accessible $\sigma$-hole regions centred on sulfur which are able to interact with Lewis bases [143]. For this series of compounds, it was shown that the enantioseparation performance of the CDMPC column is directly related to the number of fluorine atoms on the phenyl ring linked to sulfur as the electronic activators. Since the $\sigma$-hole depth of the sulfur is very sensitive to the electronic effect of bounded functional groups, it is very likely that the enantioseparation is driven by ChBs. As expected, the best result of enantioseparation was obtained with the perfluoroarylthio substituted 4,4'-bipyridine. However, the experimental results along with calculations revealed that the perfluoroaryl ring possesses a $\pi$-hole, as additional region of electron charge density depletion, that can also participate in chiral recognition. Interestingly, re- cent results on the binding properties of perfluoroarylthio substituted 4,4'-bipyridine have confirmed that this compound interacts preferentially with Lewis bases through the $\sigma$-hole [143].

\section{Conclusions}

Molecular cohesion, noncovalent interactions and supermolecules have been recognized and studied for a long time and, in the last decades, rational applications of these concepts have led to knowledge advancements in several fields such as drug discovery, molecular recognition, new materials, and crystal engineering. Also in enantioseparation science, identification and comprehension of the noncovalent forces underlying enantiodiscrimination are objectives of utmost importance in order to profile chiral recognition mechanisms. This is still a challenging task as enantioseparation is performed with polymeric selectors such as amylose- and cellulose-based derivatives. In this case, chiral recognition is a complex phenomenon involving multiple steps which are selectand adsorption (binding), diastereomeric complex formation (selection) and discrimination (function) on a multivalent polymeric platform. Moreover, nonadditive behaviour of noncovalent forces, positive and negative cooperativity, solvation and several other effects contribute to increase the level of intricacy, making extremely challenging to deconvolute the multiplicity of interactions and effects occurring during the enantioseparation process. For these purposes, multidisciplinarity and technique orthogonality were shown to be basic requirements to deconstruct enantioseparation processes occurring on the surface of polysaccharide-based selectors. In particular, X-ray analysis and spectroscopy techniques such as IR, VCD, and NMR strongly contributed to profile the high-ordered helical structure 
(a)<smiles>COC(=O)C(Br)c1c(Cl)ncc(Br)c1-c1cc(Cl)ncc1I</smiles>

29 (c)<smiles>[X]c1cnc([X])c([X])c1-c1c([X])cnc([X])c1[X]</smiles>

(b)

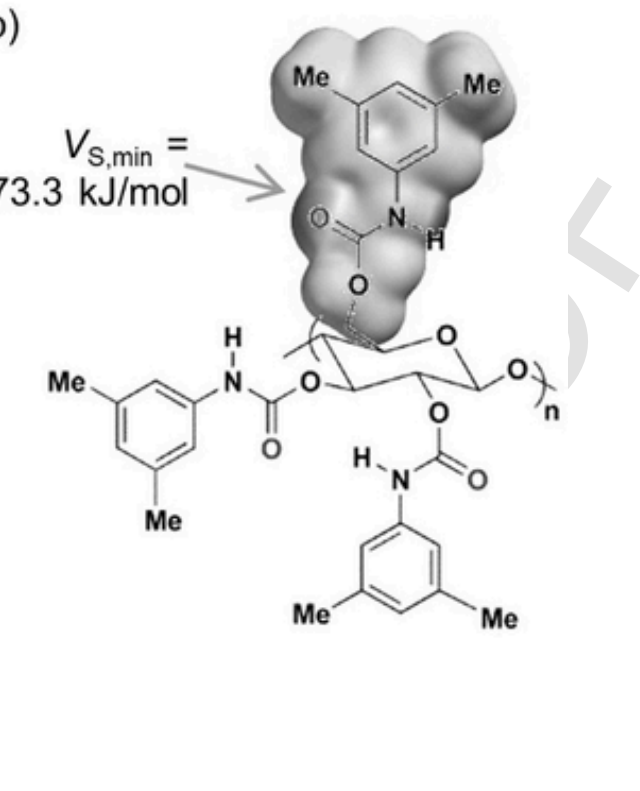

$$
\mathrm{X}=\mathrm{H}, \mathrm{Cl}, \mathrm{Br}, \mathrm{I}
$$

Fig. 21. (a) XB between iodine and carbonyl oxygen of CDMPC; (b) structures of CDMPC as XB acceptors; (c) structure of polyhalogenated 4,4'-bipyridines used as XB donor.<smiles>[Y][Y](=[Y])OC(C)C</smiles>

\section{halogen bond acceptor}

Fig. 22. Competition between $\mathrm{XB}$ and $\mathrm{HB}$ on the carbonyl oxygen.

of CTPC, CTC, CDMPC, and ADMPC. Over time integrating computer-aided approaches and experimental studies have allowed for gathering key information to decrypt chiral recognition codes, tracing future directions of the research in this field. On one hand, chemoinformatic studies and data mining have been mainly used for practical purposes such as predictions of suitable columns and appropriate operation conditions. However, the choice of suitable descriptors related to specific molecular properties can make these strategies useful for inspections at microscopic level. For this purpose, $V_{\mathrm{S}}$ analysis allows for describing shapes and stereoelectronic properties of both polymeric selector and analyte, and for correlating them with related chromatographic responses. Recently, this approach has been fruitfully used to identify and study XB in HPLC environment. On this basis, it is expected that $V_{\mathrm{S}}$ as descriptor allows the identification of new unreported noncovalent interactions as well as improvements in understanding of classical contacts such as HBs.

On the other hand, docking and MD simulations have become privileged techniques to explore chromatographic systems at microscopic level, accounting for the sensitivity of enantiorecognition to minor substituent effects. In particular, MD accounts for solvent effect and the dynamic nature of the enantioseparation phenomena. Nevertheless, despite the fact that the first MD studies of polysaccharide-based chromatographic settings date back to 1990s, actually this field is in its infancy. Indeed, so far a limited number of polysaccharide-enan- tiomer associations has been modelled by MD, some of them developed on a case-by-case basis, and general criteria to model properly polysaccharide-based selectors and simulate polysaccharide-based chromatographic systems are currently missing [24,25]. Then, computational cost of MD simulations is in general high, therefore computational choices are always the consequence of a balanced compromise between the need to obtain reliable results and approximations which depend on available computational means and complexity of the modelled chromatographic system. Several studies demonstrated that MD can help qualitatively visualize the enantiodiscrimination event and explain experimental EEO. In this regard, chromatographic phenomena of EEO reversal represent intresting experimental platforms in order to validate MD models. On the other hand, some shortcomings still remain when quantitative entities (interaction energies, geometrical parameters of noncovalent interactions) are derived from MD trajectories. In this regard, virtual experiments need to improve reliability and reproducibility of methods and approaches. Indeed, in some studies, simulation outcomes seem to nicely agree with the experimental results rather serendipitously. In other cases, no rational explanation is furnished in terms of computational details to explain the reasons why a virtual model is able or fails to describe a certain chromatographic setting.

However, the studies reported so far have contributed to profile some general guidelines: i) non-conventional enantioseparations can be fruitfully used as experimental platforms to validate MD models. Enantioseparations with large selectivity values, the use of analytes with uncommon structures and phanomena of EEO reversals are suitable for this purpose; ii) MP has to be treated explicitly in order to account for solvation effects on both CSP and analyte. The pivotal role of solvent in liquid-phase enantioseparation makes the implicitly treatment unsuitable. MD simulation performed in vacuum introduces a high level of approximation in modelling the chromatographic settings neglecting the role of MP; iii) virtual polysaccharide-based selectors should preserve as much as possible the structural identity of the real polysaccharide groove, which originates from both backbone and side chains, in terms of chain length and helical secondary structure. This aspect still remain a critical point because, in several cases, 3D-structures of poly- 


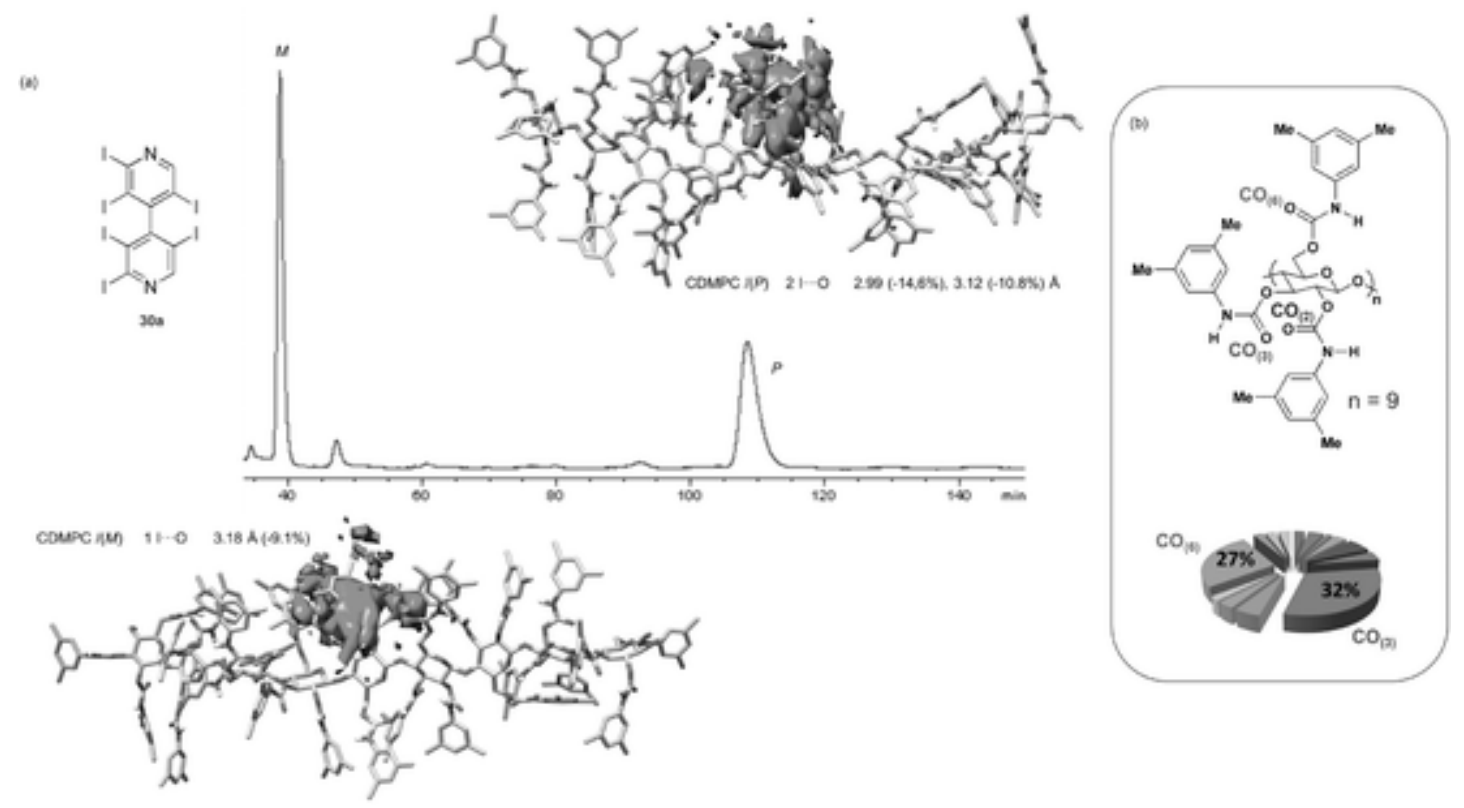

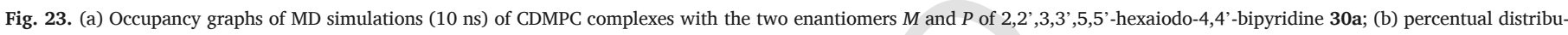
tion of the $\mathrm{C}=\underline{\mathrm{O}}$ involvement as interaction site in the course of MD simulations (10 ns). Adapted from Refs. [73,139].

accharide-derivatives have never been determined in depth, and only CTPC, CDMPC and ADMPC have been studied intensively. In this regard, it worth mentioning that in some recent published papers, a monomeric unit $[144,145]$ or short oligomer fragments $[146,147]$ have been modelled as virtual CSPs. These approaches neglect or underestimate, as a matter of fact, the impact of backbone and conformational chirality on enantioseparation, and consequently their use inevitably affects the reliability of the model

\section{CRediT authorship contribution statement}

Paola Peluso: . Victor Mamane: . Roberto Dallocchio: Writing review \& editing. Alessandro Dessì: . Sergio Cossu: .

\section{Declaration of Competing Interest}

The authors declare that they have no known competing financial interests or personal relationships that could have appeared to influence the work reported in this paper.

\section{References}

[1] J O Valderrama, The legacy of Johannes Diderik van der Waals, a hundred years after his Nobel, J. Supercritical Fluids 55 (2010) 415-420.

[2] P A Kollman, Noncovalent interactions, Acc. Chem. Res 10 (1977) 365-371.

[3] K Müller-Dethlefs, P Hobza, Noncovalent interactions: a challenge for experiment and theory, Chem. Rev 100 (2000) 143-167.

[4] J-M Lehn, Supramolecular chemistry - Scope and perspectives, Molecules, Supermolecules, and Molecular Devices (Nobel Lecture), Angew. Chem. Int. Ed. Engl. 27 (1988) 89-112.

[5] A S Mahadevi, G N Sastry, Cooperativity in noncovalent interactions, Chem. Rev 116 (2016) 2775-2825.

[6] P Politzer, J S Murray, An overview of strengths and directionalities of noncovalent interactions: $\sigma$-Holes and $\pi$-Holes, Crystals 9 (2019) 165.

[7] H J Schneider, Quantification of noncovalent interactions - promises and problems, New J. Chem 43 (2019) 15498-15512.

[8] H J Schneider, Binding mechanisms in supramolecular complexes, Angew. Chem. Int. Ed. 48 (2009) 3924-3977.

[9] M H Kolář, P Hobza, Computer modeling of halogen bonds and other $\sigma$-hole interactions, Chem. Rev. 116 (2016) 5155-5187.

[10] E Arunan, G R Desiraju, R A Klein, J Sadlej, S Scheiner, I Alkorta, D C Clary, R H Crabtree, J J Dannenberg, P Hobza, H G Kjaergaard, A C Legon, B Mennucci, D J Nesbitt, Defining the hydrogen bond: An account (IUPAC Technical Report), Pure Appl. Chem. 83 (2011) 1619-1636.

[11] G R Desiraju, P Shing Ho, L Kloo, A C Legon, R Marquardt, P Metrangolo, P Politzer, G Resnati, K Rissanen, Definition of the halogen bond (IUPAC recommendations 2013), Pure Appl. Chem. 85 (2013) 1711-1713.
[12] C B Aakeröy, D L Bryce, G R Desiraju, A Frontera, A C Legon, F Nicotra, K Rissanen, S Scheiner, G Terraneo, P Metrangolo, G Resnati, Definition of the chalcogen bond (IUPAC Recommendations 2019), Pure Appl. Chem. 91 (2019) 1889-1892.

[13] N M Maier, P Franco, W Lindner, Separation of enantiomers: needs, challenges, perspectives, J. Chromatogr. A 906 (2001) 3-33.

[14] H Lorenz, A Seidel-Morgenstern, Processes to separate enantiomers, Angew, Chem. Int. Ed. 53 (2014) 1218-1250.

[15] Y Okamoto, E Yashima, Polysaccharide derivatives for chromatographic separation of enantiomers, Angew. Chem. Int. Ed. 37 (1998) 1020-1043.

[16] B Chankvetadze, Recent developments on polysaccharide-based chiral stationary phases for liquid-phase separation of enantiomers, J. Chromatogr. A 1269 (2012) 26-51.

[17] B Chankvetadze, Polysacchride-based chiral stationary phases for enantioseparations by high-performance liquid chromatography: an overview, in: G K E Scriba (Ed.), Chiral Separations: Methods and Protocols, Methods in Molecular Biology, Vol. 1985, Springer Science + Business Media, LLC, part of Springer Nature, 2019, pp. 93-126.

[18] B Chankvetadze, Recent trends in preparation, investigation and application of polysaccharide-based chiral stationary phases for separation of enantiomers in high-performance liquid chromatography, Trends Anal. Chem. 122 (2020) 115709.

[19] K Oguni, H Oda, A Ichida, Development of chiral stationary phases consisting of polysaccharide derivatives, J. Chromatogr. A 694 (1995) 91-100.

[20] G K E Scriba, Chiral recognition in separation science - an update, J. Chromatogr. A 1467 (2016) 56-78.

[21] G K E Scriba, Chiral recognition in separation sciences. Part I: Polysaccharide and cyclodextrin selectors, Trends in Anal. Chem. 120 (2019) 115639.

[22] P Peluso, A Dessì, R Dallocchio, V Mamane, S Cossu, Recent studies of docking and molecular dynamics simulation for liquid-phase enantioseparations, Electrophoresis 40 (2019) 1881-1896.

[23] R Sardella, E Camaioni, A Macchiarulo, A Gioiello, M Marinozzi, A Carotti, Computational studies in enantioselective liquid chromatography: forty years of evolution in docking- and molecular dynamics-based simulations, Trends Anal. Chem 122 (2020) 115703.

[24] P Peluso, V Mamane, A Dessì, R Dallocchio, E Aubert, C Gatti, D Mangelings, S Cossu, Halogen bond in separation science: a critical analysis across experimental and theoretical results, J. Chromatogr. A 1616 (2020) 460788.

[25] M Lämmerhofer, Chiral recognition by enantioselective liquid chromatography: mechanisms and modern chiral stationary phases, J. Chromatogr. A 1217 (2010) 814-856.

[26] P Peluso, S Cossu, Comparative HPLC enantioseparation of thirty-six aromatic compounds on four columns of the Lux series: impact of substituents, shapes and electronic properties, Chirality 25 (2013) 709-718.

[27] P Peluso, V Mamane, E Aubert, A Dessì, R Dallocchio, A Dore, P Pale, S Cossu, Insights into halogen bond-driven enantioseparations, J. Chromatogr. A 1467 (2016) 228-238.

[28] Y Yamada, K Ohyama, G Onodera, M Kuriyama, N Kishikawa, N Kuroda, Molecular-shape selectivity by naphthalimido-modified silica stationary phases: Insight into the substituents effect of naphthalene on shape recognition and $\pi-\pi$ interactions via electrostatic potential, J. Chromatogr. A 1425 (2015) 173-179. 
[29] Y Okamoto, M Kawashima, K Hatada, Useful chiral packing materials for high-performance liquid chromatographic resolution of enantiomers: phenylcarbamates of polysaccharides coated on silica gel, J. Am. Chem. Soc. 106 (1984) 5357-5359.

[30] Y Okamoto, M Kawashima, K Hatada XI, Controlled chiral recognition of cellulose triphenylcarbamate derivatives supported on silica gel, J. Chromatogr 363 (1986) 173-186.

[31] Y Okamoto, R Aburatani, K Hatada, Chromatographic chiral resolution XIV. Cellulose tribenzoate derivatives as chiral stationary phases for high-performance liquid chromatography, J. Chromatogr 389 (1987) 95-102.

[32] Y Okamoto, R Aburatani, T Fukumoto, K Hatada, Useful chiral stationary phases for HPLC. Amylose tris(3,5-dimethylphenylcarbamate) and tris(3,5-dichlorophenylcarbamate) supported on silica gel, Chem. Lett. (1987) $1857-1860$.

[33] Y Okamoto, R Aburatani, Y Kaida, K Hatada, Direct optical resolution of carboxylic acids by chiral HPLC on tris(3,5-dimethylphenylcarbamate)s of cellulose and amylose, Chem. Lett. (1988) 1125-1128.

[34] B Chankvetadze, E Yashima, Y Okamoto, Chloromethylphenylcarbamate derivatives of cellulose as chiral stationary phases for high-performance liquid chromatography, J. Chromatogr. A 670 (1994) 39-49.

[35] B Chankvetadze, E Yashima, Y Okamoto, dimethyl-, dichloro- and chloromethylphenylcarbamates of amylose as chiral stationary phases for high-performance liquid chromatography, J. Chromatogr. A 694 (1995) 101-109.

[36] B Chankvetadze, L Chankvetadze, S Sidamonidze, E Kasashima, E Yashima, Y Okamoto, 3-Fluoro-, 3-chloro- and 3-bromo-5-methylphenylcarbamates of cellulose and amylose as chiral stationary phases for high-performance liquid-chromatographic enantioseparation, J. Chromatogr. A 787 (1997) 67-77.

[37] C Yamamoto, T Hayashi, Y Okamoto, High-performance liquid chromatographic enantioseparation using chitin carbamate derivatives as chiral stationary phases, J. Chromatogr. A 1021 (2003) 83-91.

[38] J Zhang, X C Wang, W Chen, Z W Bai, Synthesis of substituted phenylcarbamates of N-cyclobutyl formylated chitosan and their application as chiral selectors in enantioseparation, Analyst 141 (2016) 4470-4480.

[39] Y K Ye, S Bai, S Vyas, M J Wirth, NMR and computational studies of chiral discrimination by amylose tris(3,5-dimethylphenylcarbamate), J. Phys. Chem. B 111 (2007) 1189-1198.

[40] E F Pettersen, T D Goddard, C C Huang, G S Couch, D M Greenblatt, E C Meng, T E Ferrin, UCSF Chimera - a visualization system for exploratory research and analysis, J. Comput. Chem. 25 (2004) 1605-1612.

[41] W Piyatheerawong, Y Iwasaki, T Yamane, Direct separation of regio- and enantiomeric isomers of diacylglycerols by a tandem column high-performance liquid chromatography, J. Chromatogr. A 1068 (2005) 243-248.

[42] S Niedermeier, I Matarashvili, B Chankvetadze, G K E Scriba, Simultaneous determination of dextromepromazine and related substances 2-methoxyphenothiazine and levomepromazine sulfoxide in levomepromazine on a cellulose tris(4-methylbenzoate) chiral column, J. Pharm. Biomed. Anal. 158 (2018) 294-299.

[43] F Ianni, F Blasi, D Giusepponi, A Coletti, F Galli, B Chankvetadze, R Galarini, R Sardella, Liquid chromatography separation of $\alpha$ - and $\gamma$-linolenic acid positional isomers with a stationary phase based on covalently immobilized cellulose tris(3,5-dichlorophenylcarbamate), J. Chromatogr. A 1609 (2020) 460461.

[44] E R Francotte, Polysaccharide derivatives as unique chiral selectors for enantioselective chromatography, Chimia 71 (2017) 430-450.

[45] J Shen, Y Okamoto, Efficient separation of enantiomers using stereoregular chiral polymers, Chem. Rev. 116 (2016) 1094-1138.

[46] A Berthod, Chiral recognition mechanisms in enantiomers separations: a general view, in: A Berthod (Ed.), Chiral Recognition in Separation Methods Mechanisms and Applications, Springer-Verlag, Berlin Heidelberg, 2010, pp. 6-8.

[47] J C Lang, D W Armstrong, Chiral surfaces: the many faces of chiral recognition, Curr. Opin. Colloid Interface Sci. 32 (2017) 94-107.

[48] C Bissantz, B Kuhn, M Stahl, A medicinal chemist's guide to molecular interactions, J. Med. Chem 53 (2010) 5061-5084.

[49] P Peluso, V Mamane, E Aubert, S Cossu, Insights into the impact of shape and electronic properties on the enantioseparation of polyhalogenated 4,4'-bipyridines on polysaccharide-type selectors. Evidence of stereoselective halogen bonding interactions, J. Chromatogr. A 1345 (2014) 182-192.

[50] P Politzer, P Lane, M C Concha, Y Ma, J S Murray, An overview of halogen bonding, J. Mol. Model. 13 (2007) 305-311.

[51] J S Murray, P Politzer, Molecular electrostatic potentials and noncovalent interactions, WIREs Comput. Mol. Sci. 7 (2017) e1326.

[52] P Peluso, V Mamane, E Aubert, S Cossu, Optimization of the HPLC enantioseparation of 3,3'-dibromo-5,5'-disubstituted-4,4'-bipyridines using immobilized polysaccharide-based chiral stationary phases, J. Sep. Sci. 36 (2013) 2993-3003.

[53] P Peluso, V Mamane, E Aubert, S Cossu, High-performance liquid chromatography enantioseparation of polyhalogenated 4,4'-bipyridines on polysaccharide-based chiral stationary phases under multimodal elution, J. Sep. Sci. 37 (2014) 2481-2489.

[54] K G Lynam, R W Stringham, Chiral separations on polysaccharide stationary phases using polar organic mobile phases, Chirality 18 (2006) 1-9.

[55] R Ferretti, A Mai, B Gallinella, L Zanitti, S Valente, R Cirilli, Application of 3 $\mu \mathrm{m}$ particle-based amylose-derived chiral stationary phases for the enantioseparation of potential histone deacetylase inhibitors, J. Chromatogr. A 1218 (2011) 8394-8398.
[56] T Zhang, P Franco, Analytical and preparative potential of immobilized polysaccharide-derived chiral stationary phases, in: G Subramanian (Ed.), Chiral Separation Techniques - A Practical Approach, 3rd ed, Wiley-VCH, Weinheim, Germany, 2006, pp. 99-134.

[57] T Zhang, D Nguyen, P Franco, Reversed-phase screening strategies for liquid chromatography on polysaccharide-derived chiral stationary phases, J. Chromatogr. A 1217 (2010) 1048-1055.

[58] L Peng, S Jayapalan, B Chankvetadze, T Farkas, Reversed-phase chiral HPLC and LC/MS analysis with tris(chloromethylphenylcarbamate) derivatives of cellulose and amylose as chiral stationary phases, J. Chromatogr. A 1217 (2010) 6942-6955.

[59] B Chankvetadze, C Yamamoto, Y Okamoto, Enantioseparation of selected chiral sulfoxides using polysaccharide-type chiral stationary phases and polar organic, polar aqueous-organic and normal-phase eluents, J. Chromatogr. A 922 (2001) 127-137.

[60] S Materazzo, S Carradori, R Ferretti, B Gallinella, D Secci, R Cirilli, Effect of the water content on the retention and enantioselectivity of albendazole and fenbendazole sulfoxides using amylose-based chiral stationary phases in organic-aqueous conditions, J. Chromatogr. A 1327 (2014) 73-79.

[61] I Matarashvili, D Ghughunishvili, L Chankvetadze, N Takaishvili, T Khatiashvili, M Tsintsadze, T Farkas, B Chankvetadze, Separation of enantiomers of chiral weak acids with polysaccharide-based chiral columns and aqueous-organic mobile phases in high-performance liquid chromatography: Typical reversed-phase behavior?, J. Chromatogr. A 1483 (2017) 86-92.

[62] M Okamoto, Reversal of elution order during the chiral separation in high-performance liquid chromatography, J. Pharm. Biomed. Anal. 27 (2002) 401-407.

[63] S Ma, S Shen, H Lee, M Eriksson, X Zeng, J Xu, K Fandrick, N Yee, C Senanayake, N Grinberg, Mechanistic studies on the chiral recognition of polysaccharide-based chiral stationary phases using liquid chromatography and vibrational circular dichroism. Reversal of elution order of $\mathrm{N}$-substituted alpha-methyl phenylalanine esters, J. Chromatogr. A 1216 (2009) 3784-3793.

[64] I Matarashvili, L Chankvetadze, S Fanali, T Farkas, B Chankvetadze, HPLC separation of enantiomers of chiral arylpropionic acid derivatives using polysaccharide-based chiral columns and normal-phase eluents with emphasis on elution order, J. Sep. Sci. 36 (2013) 140-147.

[65] I Matarashvili, G Kobidze, A Chelidze, G Dolidze, N Beridze, G Jibuti, T Farkas, B Chankvetadze, The effect of temperature on the separation of enantiomers with coated and covalently immobilized polysaccharide-based chiral stationary phases, J. Chromatogr. A 1599 (2019) 172-179.

[66] P Peluso, D Fabbri, M A Dettori, G Delogu, V Zambrano, S Cossu, High-performance liquid chromatographic enantioseparation of atropisomeric biphenyls on seven chiral stationary phases, Curr. Org. Chem. 15 (2011) 1208-1229.

[67] P Peluso, V Mamane, E Aubert, S Cossu, High-performance liquid chromatography enantioseparation of atropisomeric 4,4'-bipyridines on polysaccharide-type chiral stationary phases: Impact of substituents and electronic properties, J. Chromatogr. A 1251 (2012) 91-100.

[68] L D Asnin, M V Stepanova, Van't Hoff analysis in chiral chromatography, J. Sep. Sci. 41 (2018) 1319-1337.

[69] A Sepsey, E Horváth, M Catani, A Felinger, The correctness of van't Hoff plots in chiral and achiral chromatography, J. Chromatogr. A 1611 (2020) 460594.

[70] C Yamamoto, E Yashima, Y Okamoto, Structural analysis of amylose tris(3,5-dimethylphenylcarbamate) by NMR relevant to its chiral recognition mechanism in HPLC, J. Am. Chem. Soc. 124 (2002) 12583-12589.

[71] R B Kasat, Y Zvinevich, H W Hillhouse, K T Thomson, N-H L Wang, E I Franses, Direct probing of sorbent-solvent interactions for amylose tris(3,5-methylphenylcarbamate) using infrared spectroscopy, X-ray diffraction, solid state NMR, and DFT modeling, J. Phys. Chem. B 110 (2006) 14114-14122.

[72] E Yashima, M Yamada, Y Kaida, Y Okamoto, Computational studies on chiral discrimination mechanism of cellulose trisphenylcarbamate, J. Chromatogr. A 694 (1995) 347-354.

[73] R Dallocchio, A Dessì, M Solinas, A Arras, S Cossu, E Aubert, V Mamane, P Peluso, Halogen bond in high-performance liquid chromatographyenantioseparations: description, features and modelling, J. Chromatogr. A 1563 (2018) 71-81.

[74] C R Mitchell, N J Benz, S Zhang, Comparison of the factors that contribute to retention on immobilized polysaccharide-based chiral stationary phases and macrocyclic glycopeptide chiral stationary phases with the Abraham model, J. Chromatogr. B 875 (2008) 65-71.

[75] A Del Rio, Exploring enantioselective molecular recognition mechanisms with chemoinformatic techniques, J. Sep. Sci. 32 (2009) 1566-1584.

[76] G Guenegou C.West, Y Zhang, L Morin-Allory, Insights into chiral recognition mechanisms in supercritical fluid chromatography. II. Factors contributing to enantiomer separation on tris-(3,5-dimethylphenylcarbamate) of amylose and cellulose stationary phases, J. Chromatogr. A 1218 (2011) 2033-2057.

[77] P K Weiner, R Langridge, J M Blaney, R Schaefer, P A Kollman, Electrostatic potential molecular surfaces, Proc. Natl. Acad. Sci. USA 79 (1982) 3754-3758.

[78] Y Li, D Liu, P Wang, Z Zhou, Computational study of enantioseparation by amylose tris(3,5-dimethylphenylcarbamate)-based chiral stationary phases, J. Sep. Sci. 33 (2010) 3245-3255.

[79] B-H Kim, S U Lee, D C Moon, Chiral recognition of $N$-phthaloyl, $N$-tetrachlorophthaloyl, and $N$-naphthaloyl $\alpha$-amino acids and their esters on polysaccharide-derived chiral stationary phases, Chirality 24 (2012) 1037-1046. 
[80] G Hu, M Huang, C Luo, Q Wang, J-W Zou, Interactions between pyrazole derived enantiomers and Chiralcel OJ: Prediction of enantiomer absolute configurations and elution order by molecular dynamics simulations, J. Mol. Graph. Model. 66 (2016) 123-132.

[81] T Brick, J H Stenlid, The molecular surface property approach: a guide to chemical interactions in chemistry, medicine, and material science, Adv. Theory Simul. 2 (2019) 1800149.

[82] A Del Rio, P Piras, C Roussel, Data mining and enantiophore studies on chiral stationary phases used in HPLC separation, Chirality 17 (2006) S74-S83.

[83] K E Riley, K-A Tran, P Lane, J S Murray, P Politzer, Comparative analysis of electrostatic potential maxima and minima on molecular surfaces, as determined by three methods and a variety of basis sets, J. Comput. Sci. 17 (2016) 273-278.

[84] D T Stanton, P C Jurs, Development and use of charged partial surface area structural descriptors in computer-assisted quantitative structure-property relationship studies, Anal. Chem. 62 (1990) 2323-2329.

[85] T D Booth, W J Lough, M Saeed, T A G Noctor, I W Wainer, Enantioselective separation of enantiomeric amides on three amylose-based chiral stationary phases: effects of backbone and carbamate side chain chiralities, Chirality 9 (1997) 173-177.

[86] T D Booth, K Azzaoui, I W Wainer, Prediction of chiral chromatographic separations using combined multivariate regression and neural networks, Anal. Chem. 69 (1997) 3879-3883.

[87] S Schefzick, M Lämmerhofer, W Lindner, K B Lipkowitz, M Jalaie, Comparative molecular field analysis of quinine derivatives used as chiral selectors in liquid chromatography: 3D QSAR for the purposes of molecular design of chiral stationary phases, Chirality 12 (2000) 742-750.

[88] W M F Fabian, W Stampfer, M Mazur, G Uray, Modeling the chromatographic enantioseparation of aryl- and hetarylcarbinols on ULMO, a brush-type chiral stationary phase, by 3D-QSAR techniques, Chirality 15 (2003) 271-275.

[89] B Natalini, R Sardella, A Macchiarulo, S Natalini, R Pellicciari, (S)-(-)- $\alpha, \alpha-\operatorname{Di}(2-n a p h t h y l)-2-p y r r o l i d i n e m e t h a n o l$, a useful tool to study the recognition mechanism in chiral ligand-exchange chromatography, J. Sep. Sci. 30 (2007) 21-27.

[90] A Ichida, T Shibata, I Okamoto, Y Yuki, H Namikoshi, Y Toga, Resolution of enantiomers by HPLC on cellulose derivatives, Chromatographia 19 (1984) 280-284.

[91] U Vogt, P Zugenmaier, Structural models for some liquid crystalline cellulose derivatives, Ber. Bunsenges. Phys. Chem 89 (1985) 1217-1224.

[92] E Yashima, C Yamamoto, Y Okamoto, NMR studies of chiral discrimination relevant to the liquid chromatographic enantioseparation by a cellulose phenylcarbamate derivative, J. Am. Chen. Soc. 118 (1996) 4036-4048.

[93] J Danhelka, M Netopilík, M Bohdanecky, Solution, properties and chain conformation characteristics of cellulose tricarbanilate, J. Polym. Sci. Part B: Polym. Phys. 25 (1987) 1801-1815.

[94] C Yamamoto, E Yashima, Y Okamoto, Computational studies on chiral discrimination mechanism of phenylcarbamate derivatives of cellulose, Bull. Chem. Soc. Jpn. 72 (1999) 1815-1825.

[95] P Zugenmaier, H Steinmeier, Conformation of some amylose triesters: the influence of side groups, Polymer 27 (1986) 1601-1608.

[96] R M Wenslow Jr., T Wang, Solid-state NMR characterization of amylose tris(3,5-dimethylphenylcarbamate) chiral stationary-phase structure as a function of mobile-phase composition, Anal. Chem. 73 (2001) 4190-4195.

[97] T D Booth, I W Wainer, Investigation of the enantioselective separations of $\alpha$-alkylarylcarboxylic acids on an amylose tris(3,5-dimethylphenylcarbamate) chiral stationary phase using quantitative structure-enantioselective retention relationships. Identification of a conformationally driven chiral recognition mechanism, J. Chromatogr. A 737 (1996) 157-169.

[98] R B Kasat, N-H L Wang, E I Franses, Effects of backbone and side chain on the molecular environments of chiral cavities in polysaccharide-based biopolymers, Biomacromolecules 8 (2007) 1676-1685.

[99] H Steinmeier, P Zugenmaier, Homogeneous and heterogeneous cellulose triesters and a cellulose triurethane: synthesis and structural investigations of the crystalline state, Carbohydr. Res. 164 (1987) 97-105.

[100] E Francotte, R M Wolf, Benzoyl cellulose beads in the pure polymeric form as a new powerful sorbent for the chromatographic resolution of racemates, Chirality 3 (1991) 43-55.

[101] T O'Brien, L Crocker, R Thompson, K Thompson, P H Toma, D A Conlon, B Feibush, C Moeder, G Bicker, N Grinberg, Mechanistic aspects of chiral discrimination on modified cellulose, Anal. Chem. 69 (1997) 1999-2007.

[102] C Yamamoto, K Yamada, K Motoya, Y Kamiya, M Kamigaito, Y Okamoto, T Aratani, Preparation of HPLC chiral packing materials using cellulose tris(4-methylbenzoate) for the separation of chrysanthemate isomers, J. Polym. Sci., Part A: Polym. Chem. 44 (2006) 5087-5097.

[103] R B Kasat, C Y Chin, K T Thomson, E I Franses, N-H L Wang, Interpretation of chromatographic retentions of simple solutes with an amylose-based sorbent using infrared spectroscopy and DFT modeling, Adsorption 12 (2006) 405-416.

[104] B Zhao, P A Oroskar, X Wang, D House, A Oroskar, A Oroskar, C J Jameson, S Murad, The composition of the mobile phase affects the dynamic chiral recognition of drug molecules by the chiral stationary phase, Langmuir 33 (2017) 11246-11256.

[105] S Ma, S Shen, H Lee, N Yee, C Senanayake, L A Nafie, N Grinberg, Vibrational circular dichroism of amylose carbamate: structure and solvent-induced conformational changes, Tetrahedron: Asymmetry 19 (2008) 2111-2114.
[106] S Horváth, G Németh, Hysteresis of retention and enantioselectivity on amylose tris(3,5-dimethylphenylcarbamate) chiral stationary phases in mixtures of 2-propanol and methanol, J. Chromatogr. A 1568 (2018) 149-159.

[107] K Ogura, Y Miyachi, H Soboue, S Nakamura, Infrared spectroscopic studies of polymer transitions. 4) A second-order transition of cellulose triacetate at the vicinity of $30^{\circ} \mathrm{C}$, Die Makromol. Chem. 116 (1975) 1173-1178.

[108] A K Gupta, E Marchal, W Burchard, Effect of temperature on the flexibility of cellulose tricarbanilate in dioxane and ethyl acetate by dielectric measurements, Macromolecules 8 (1975) 843-849.

[109] F Wang, T O'Brien, T Dowling, G Bicker, J Wyvratt, Unusual effect of column temperature on chromatographic enantioseparation of dihydropyrimidinone acid and methyl ester on amylose chiral stationary phase, J. Chromatogr. A 958 (2002) 69-77.

[110] F Wang, R M Wenslow Jr., T M Dowling, K T Mueller, I Santos, J M Wyvratt, Characterization of a thermally induced irreversible conformational transition of amylose tris(3,5-dimethylphenylcarbamate) chiral stationary phase in enantioseparation of dihydropyrimidinone acid by quasi-equilibrated liquid chromatography and solid-state NMR, Anal. Chem. 75 (2003) 5877-5885.

[111] B Feibush, Chiral separation of enantiomers via selector/selectand hydrogen bondings, Chirality 10 (1998) 382-395.

[112] A Del Rio, J M Hayes, M Stein, P Piras, Roussel C, Theoretical reassessment of Whelk-O1 as an enantioselective receptor for 1-(4-halogeno-phenyl)-1-ethylamine derivatives, Chirality 16 (2004) S1-S11.

[113] A Del Rio, J Gasteiger, Simple method for the prediction of the separation of racemates with high-performance liquid chromatography on Whelk-O1 chiral stationary phase, J. Chromatogr. A 1185 (2008) 49-58.

[114] C E Dalgliesh, The optical resolution of aromatic amino-acids on paper chromatograms, J. Chem. Soc. 132 (1952) 3940-3942.

[115] I W Wainer, Proposal for the classification of high-performance-liquid chromatographic chiral stationary phases: how to choose the right column, Trends Anal. Chem. 6 (1987) 125-134.

[116] T D Booth, D Wahnon, I W Wainer, Is chiral recognition a three-point process?, Chirality 9 (1997) 96-98.

[117] K B Lipkowitz, Atomistic modeling of enantioselection in chromatography, J. Chromatogr. A 906 (2001) 417-442.

[118] P Camilleri, J A Murphy, M R Saunders, C J Thorpe, Molecular modelling studies and the chromatographic behaviour of oxiracetam and some closely related molecules, J. Comput. -Aided Mol. Des. 5 (1991) 277-284.

[119] E Yashima, M Yamada, Y Okamoto, An NMR study of chiral recognition relevant to the liquid chromatographic separation of enantiomers by a cellulose derivative, Chem. Lett. (1994) 579-582.

[120] E Yashima, M Yamada, C Yamamoto, N Nakashima, Y Okamoto, Chromatographic enantioseparation and chiral discrimination in NMR by trisphenylcarbamate derivatives of cellulose, amylose, oligosaccharides, and cyclodextrins, Enantiomer 2 (1997) 225-240.

[121] R B Kasat, S Y Wee, J X Loh, N-H L Wang, E I Franses, Effect of solute molecular structure on its enantioresolution on cellulose tris(3,5-dimethylphenylcarbamate), J. Chromatogr. B 875 (2008) 81-92.

[122] R B Kasat, N-H L Wang, E I Franses, Experimental probing and modeling of key sorbent-solute interactions of norephedrine enantiomers with polysaccharide-based chiral stationary phases, J. Chromatogr. A 1190 (2008) 110-119.

[123] S Ma, H-W Tsui, E Spinelli, C A Busacca, E I Franses, N-H L Wang, L Wu, H Lee, C Senanayake, N Yee, N Gonella, K Fandrick, N Grinberg, Insights into chromatographic enantiomeric separation of allenes on cellulose carbamate stationary phase, J. Chromatogr. A 1362 (2014) 119-128.

[124] Y Li, D Liu, P Wang, Z Zhou, Computational study of enantioseparation by amylose tris(3,5-dimethylphenylcarbamate)-based chiral stationary phase, J. Sep. Sci. 33 (2010) 3245-3255.

[125] X Wang, C J Jameson, S Murad, Modeling enantiomeric separations as an interfacial process using amylose tris(3,5-dimethyl carbamate) (ADMPC) polymers coated on amorphous silica, Langmuir 36 (2020) 1113-1124.

[126] X Wang, D H House, P A Oroskar, A Oroskar, A Oroskar, C J Jameson, S Murad, Molecular dynamics simulations of the chiral recognition mechanism for a polysaccharide chiral stationary phase in enantiomeric chromatographic separations, Mol. Phys. 117 (2019) 3569-3588.

[127] S Alcaro, A Bolasco, R Cirilli, R Ferretti, R Fioravanti, F Ortuso, Computer-aided molecular design of asymmetric pyrazole derivatives with exceptional enantioselective recognition toward the Chiralcel OJ-H stationary phase, J. Chem. Inf. Model. 52 (2012) 649-654.

[128] F Ortuso, S Alcaro, S Menta, R Fioravanti, R Cirilli, A chromatographic and computational study on the driving force operating in the exceptionally large enantioseparation of

N-thiocarbamoyl-3-(4'-biphenyl)-5-phenyl-4,5-dihydro-(1H) pyrazole on a 4-methylbenzoate cellulose-based chiral stationary phase, J. Chromatogr. A 1324 (2014) 71-77.

[129] B Chankvetadze, C Yamamoto, Y Okamoto, Extremely high enantiomer recognition in HPLC separation of racemic 2-(benzylsulfinyl)benzamide using cellulose tris(3,5-dichlorophenylcarbamate), Chem. Lett. (2000) 1176-1177.

[130] C West, M-L Konjaria, N Shashviashvili, E Lemassona, P Bonnet, R Kakava, A Volonterio, B Chankvetadze, Enantioseparation of novel chiral sulfoxides on chlorinated polysaccharide stationary phases in supercritical fluid chromatography, J. Chromatogr. A 1499 (2017) 174-182.

[131] T Khatiashvili, R Kakava, I Matarashvili, H Tabani, C Fanali, A Volonterio, T Farkas, B Chankvetadze, Separation of enantiomers of selected chiral sulfoxides with cellulose tris(4-chloro-3-methylphenylcarbamate)-based chiral columns in high-performance liquid chromatography with very high separation factor, J. Chromatogr. A 1545 (2018) 59-66. 
[132] Z Shedania, R Kakava, A Volonterio, T Farkas, B Chankvetadze, Separation of enantiomers of chiral sulfoxides in high-performance liquid chromatography with cellulose-based chiral selectors using methanol and methanol-water mixtures as mobile phases, J. Chromatogr. A 1557 (2018) 62-74.

[133] Z Shedania, R Kakava, A Volonterio, T Farkas, B Chankvetadze, Separation of enantiomers of chiral sulfoxides in high-performance liquid chromatography with cellulose-based chiral selectors using acetonitrile and acetonitrile-water mixtures as mobile phases, J. Chromatogr. A 1609 (2020) 460445.

[134] S Carradori, D Secci, P Guglielmi, M Pierini, R Cirilli, High-performance liquid chromatography enantioseparation of chiral 2-(benzylsulfinyl)benzamide derivatives on cellulose tris(3,5-dichlorophenylcarbamate) chiral stationary phase, J. Chromatogr. A 1610 (2020) 460572.

[135] R Cirilli, R Ferretti, B Gallinella, L Turchetto, A Bolasco, D Secci, P Chimenti, M Pierini, V Fares, O Befani, F La Torre, Enantiomers of $\mathrm{C}_{5}$-chiral 1-acetyl-3,5-diphenyl-4,5-dihydro-(1H)-pyrazole derivatives: analytical and semipreparative HPLC separation, chiroptical properties, absolute configuration, and inhibitory activity against monoamine oxidase, Chirality 16 (2004) 625-636.

[136] B-H Kim, S U Lee, K T Kim, J-Y Lee, N H Choi, Y-K Han, J H Ok, Enantiomeric discrimination of pyrethroic acid esters on polysaccharide derived chiral stationary phases, Chirality 15 (2003) 276-283.

[137] B-H Kim, S U Lee, Enantiomer separation of a novel aminothiazolecarboxamide fungicide using polysaccharide-derived chiral stationary phases, J. Chromatogr. Sci. 43 (2005) 501-504.

[138] G Cavallo, P Metrangolo, R Milani, T Pilati, A Priimagi, G Resnati, G Terraneo, The halogen bond, Chem. Rev. 116 (2016) 2478-2601.

[139] P Peluso, V Mamane, R Dallocchio, A Dessì, R Villano, D Sanna, E Aubert, P Pale, S Cossu, Polysaccharide-based chiral stationary phases as halogen bond acceptors: a novel strategy for detection of stereoselective $\sigma$-hole bonds in solution, J. Sep. Sci. 41 (2018) 1247-1256.

[140] V Mamane, P Peluso, E Aubert, S Cossu, P Pale, Chiral hexahalogenated 4,4'-bipyridines, J. Org. Chem. 81 (2016) 4576-4587.

[141] P Peluso, C Gatti, A Dessì, R Dallocchio, R Weiss, E Aubert, P Pale, S Cossu, V Mamane, Enantioseparation of fluorinated 3-arylthio-4,4'-bipyridines: insights into chalcogen and $\pi$-hole bonds in high-performance liquid chromatography, $\mathrm{J}$. Chromatogr. A 1567 (2018) 119-129.

[142] W Wang, B Ji, Y Zhang, Chalcogen bond: A sister noncovalent bond to halogen bond, J. Phys. Chem. A 113 (2009) 8132-8135.

[143] R Weiss, E Aubert, P Peluso, S Cossu, P Pale, V Mamane, Chiral chalcogen bond donors based on the 4,4'-bipyridine scaffold, Molecules 24 (2019) 4484.

[144] C Fonseca Silva, L Guimarães, K B Borges, C S Nascimento Jr, Development and validation of an experimental and theoretical method for the chiral discrimination of dinotefuran, Chirality 32 (2020) 53-63.

[145] C Fonseca Silva, T A do Nascimento, L Guimarães, K B Borges, C S Nascimento $\mathrm{Jr}$, Elucidation of the chromatographic enantiomer elution order for praziquantel: An experimental and theoretical assessment, Chirality 32 (2020) 353-358.

[146] M Li, B Zhang, J Yu, J Wang, X Guo, Enantiomeric separation and simulation study of eight anticholinergic drugs on an immobilized polysaccharide-based chiral stationary phase by HPLC, New J. Chem. 42 (2018) 11724-11731.

[147] H Barfeii, Z Garkani-Nejad, V Saheb, Investigation of the mechanism of enantioseparation of some drug compounds by considering the mobile phase in HPLC by molecular dynamic simulation, J. Mol. Mod. 25 (2019) 297. 